\title{
Modeling Repeated M Dwarf Flaring at an Earth-like Planet in the Habitable Zone: Atmospheric Effects for an Unmagnetized Planet
}

\author{
Matt A. Tilley, ${ }^{1-3}$ Antígona Segura, ${ }^{2,4}$ Victoria Meadows, ${ }^{2,3,5}$ \\ Suzanne Hawley, ${ }^{2,3,5}$ and James Davenport ${ }^{2,6}$
}

\begin{abstract}
Understanding the impact of active $\mathrm{M}$ dwarf stars on the atmospheric equilibrium and surface conditions of a habitable zone Earth-like planet is key to assessing M dwarf planet habitability. Previous modeling of the impact of electromagnetic (EM) radiation and protons from a single large flare on an Earth-like atmosphere indicated that significant and long-term reductions in ozone were possible, but the atmosphere recovered. However, these stars more realistically exhibit frequent flaring with a distribution of different total energies and cadences. Here, we use a coupled 1D photochemical and radiative-convective model to investigate the effects of repeated flaring on the photochemistry and surface UV of an Earth-like planet unprotected by an intrinsic magnetic field. As input, we use time-resolved flare spectra obtained for the dM3 star AD Leonis, combined with flare occurrence frequencies and total energies (typically $10^{30.5}$ to $10^{34} \mathrm{erg}$ ) from the 4-year Kepler light curve for the dM4 flare star GJ1243, with varied proton event impact frequency. Our model results show that repeated EM-only flares have little effect on the ozone column depth but that multiple proton events can rapidly destroy the ozone column. Combining the realistic flare and proton event frequencies with nominal CME/SEP geometries, we find the ozone column for an Earth-like planet can be depleted by $94 \%$ in 10 years, with a downward trend that makes recovery unlikely and suggests further destruction. For more extreme stellar inputs, $\mathrm{O}_{3}$ depletion allows a constant $\sim 0.1-1 \mathrm{~W} \mathrm{~m}^{-2}$ of UVC at the planet's surface, which is likely detrimental to organic complexity. Our results suggest that active $\mathrm{M}$ dwarf hosts may comprehensively destroy ozone shields and subject the surface of magnetically unprotected Earth-like planets to long-term radiation that can damage complex organic structures. However, this does not preclude habitability, as a safe haven for life could still exist below an ocean surface. Key Words: M dwarf-Flares-Stellar activity-Habitable zone-Planetary atmospheres-Magnetic field. Astrobiology 19, 64-86.
\end{abstract}

\section{Introduction}

W HETHER M DWARF PLANETS are good targets in the search for life is still an open problem of high complexity. There exist several factors that make such planets promising targets-primarily, detectability and abundance. Rocky planets are easier to detect around these low-mass stars $\left(0.5-0.08 M_{\odot}\right)$ with current techniques and instruments. Red dwarfs are abundant in the solar neighborhood, they spend $\sim 10^{10}$ years in the main sequence, and biosignatures like $\mathrm{N}_{2} \mathrm{O}$ and $\mathrm{CH}_{4}$ may be more abundant and potentially easier to detect (Segura et al., 2005; see also reviews by Scalo et al., 2007; Tarter et al., 2007; Shields et al., 2016; Meadows et al., 2018a). The abundance of M dwarf hosts, and therefore

\footnotetext{
${ }^{1}$ Department of Earth and Space Sciences, University of Washington, Seattle, Washington, USA.

${ }^{2}$ NASA Astrobiology Institute-Virtual Planetary Laboratory Lead Team, USA.

${ }^{3}$ Astrobiology Program, University of Washington, Seattle, Washington, USA.

${ }^{4}$ Instituto de Ciencias Nucleares, Universidad Nacional Autónoma de México, México.

${ }^{5}$ Department of Astronomy, University of Washington, Seattle, Washington, USA.

${ }^{6}$ Department of Physics and Astronomy, Western Washington University, Bellingham, Washington, USA.
}

(C) Matt A. Tilley et al., 2018; Published by Mary Ann Liebert, Inc. This Open Access article is distributed under the terms of the Creative Commons Attribution Noncommercial License (http://creativecommons.org/licenses/by-nc/4.0/) which permits any noncommercial use, distribution, and reproduction in any medium, provided the original author(s) and the source are credited. 
potentially habitable planets, is beneficial since planets orbiting these hosts in their proximal habitable zones encounter conditions that could make habitability challenging.

Three-dimensional planetary atmospheric models have dispelled concerns of atmospheric collapse by extreme temperature differences between the illuminated and dark hemispheres (e.g., Dole, 1964) for tidally locked planets orbiting M dwarf hosts in the habitable zone; the models applied to this problem showed that atmospheric circulation can distribute the stellar energy, lowering the temperature difference (e.g., Joshi et al., 1997; Joshi, 2003; Turbet et al., 2016). Active M dwarf host stars, however, exhibit magnetic activity originated by the interaction of their atmospheres with their magnetic fields driven by their mostly or totally convective interiors. One consequence of magnetic activity are flares, unpredictable releases of energy that range from "microflares" ( $\sim 10^{29} \mathrm{erg}$ as measured in the U band, 3320-3980 $\AA$ ) to high-energy flares with total energies as large as $10^{34} \mathrm{erg}$ (e.g., Hawley and Pettersen, 1991; Hawley et al., 2014). Since the habitable zone around these stellar hosts is located at a fraction of an astronomical unit, the energy flux from these events that impact potentially habitable worlds is increased by at least an order of magnitude compared to Earth.

Active $\mathrm{M}$ dwarfs exhibit flare activity with widely varying flare energies and frequencies but generally following a power-law probability distribution (e.g., Hilton, 2011; Hawley et al., 2014). The most active of these dM3-dM5 stars have been observed to produce dozens of flares per day with total energies $\geq 10^{30}$ erg and potentially catastrophic events like the Great Flare of AD Leonis at $10^{34}$ erg (Hawley and Pettersen, 1991) at a frequency of once per month. Compare this to average solar activity with the highest frequency of a single event per day of energy $\sim 10^{27} \mathrm{erg}$, up to approximately one $10^{31}$ erg event per year (e.g., Crosby et al., 1993). One of the largest observed solar flare events, known as the Carrington event of 1859, had an estimated total energy of $\sim 10^{32}$ erg (e.g., Cliver and Dietrich, 2013), a full 2 orders of magnitude lower than the most energetic events observed on active $\mathrm{M}$ dwarfs.

There are two aspects of flare events that could negatively impact the habitability of a planet by altering the atmospheric composition: increase in electromagnetic flux, and an eruption of energized charged particles. The energy injected by a flare into the stellar atmosphere results in a rise and peak of photometric brightness, known as the impulsive phase. A gradual flare decay phase follows, where the energy input decreases until the star slowly returns to quiescent state. During flare events, the luminosity of the star in the X-ray, $\mathrm{UV}$, and visible increases up to 3 orders of magnitude (Scalo et al., 2007), which can photochemically alter the upper atmosphere of a planet (Segura et al., 2010). For a planet that does not have atmospheric constituents (e.g., $\left.\mathrm{O}_{2}, \mathrm{O}_{3}, \mathrm{CO}_{2}\right)$ to absorb this short-wavelength radiation, the surface could be regularly irradiated.

The second impactful aspect of flare events involves stellar energetic particles (SEPs) that can be accelerated during the impulsive phase of flares. Our knowledge of these events comes only from solar correlations, as we have no method by which they can be remotely observed. Their effect on the atmosphere of a habitable planet likely depends on several factors: the particle energies, presence and orientation of a planetary magnetic field, and the chemical composition of the atmosphere. The probability of a proton event being associated with a stellar flare is dependent on the flare energy, with solar microflares $\left(\leq 10^{27} \mathrm{erg}\right)$ only rarely producing weak particle events (e.g., Hudson, 2011). The chance for a large flux of energetic particles reaches a near certainty for flares $\sim 10^{28.3}$ erg (GOES class X2) and above (e.g., Yashiro et al., 2006; Dierckxsens et al., 2015). While not every proton event will hit a potentially habitable planet due to the combined geometry of the active regions of the star and the planetary orbit (e.g., Khodachenko et al., 2007), M dwarf planets we consider are under significant threat. These planets orbit at a fraction of an astronomical unit, and the stellar hosts exhibit high-frequency, highenergy flare events, indicating the effects of multiple impacting proton events could be significant.

Studies on the effects of space weather on habitability have become crucial with the discovery of planets such as Proxima Centauri b (Anglada-Escudé et al., 2016) and the TRAPPIST-1 (Gillon et al., 2016, 2017) systems' multiple habitable zone planets. Planetary atmospheric escape can be caused by XUV radiation that heats the planet's exosphere, driving a hydrodynamic wind that carries away the planetary atmosphere (e.g., Luger and Barnes, 2015); non-thermal processes such as the increased polar wind and ion pickup escape can potentially remove vast quantities of heavy ions such as $\mathrm{N}^{+}$ and $\mathrm{O}^{+}$from the atmosphere (e.g., Ribas et al., 2016; Airapetian et al., 2017; Garcia-Sage et al., 2017); increased forcing of the magnetosphere by either steady state or transient stellar wind events can also be impactful for atmospheric loss and the subsequent atmospheric outflow (e.g., Garraffo et al., 2016, 2017; Dong et al., 2018). However, while many papers have focused on atmospheric loss for planets orbiting $M$ dwarfs, less research has focused on the effects of space weather events on the chemical evolution of planets that retain their atmospheres, and how these processes impact habitability.

Segura et al. (2010) performed the first study to explore the effect of a single, high-energy flare (and associated SEPs) on the atmospheric chemistry of a habitable planet; their study investigated the impact of an event equivalent to the 1985 April 12 flare from the M dwarf AD Leonis (AD Leo) (Hawley and Pettersen, 1991) on an Earth-like planet located within its habitable zone. The planetary atmosphere had a similar composition to that of present Earth $\left(0.21 \mathrm{O}_{2}\right.$, 1 bar surface pressure) and received the same integrated stellar flux as our planet. They used a 1D photochemical model coupled to a $1 \mathrm{D}$ radiative/convective model to simulate the effects of both UV radiation and protons on the atmospheric chemistry of a hypothetical habitable planet. Observations available for this flare included UV spectroscopy (1150-3100 ̊) and optical spectroscopy (3560-4440 ̊), but no proton event data are available. To include particles, Segura et al. (2010) used a relationship found for solar X-ray flare intensity and proton fluxes (Belov et al., 2005). X-ray flare intensities were calculated via the Neupert effect, an empirical relation between the flare energy emitted in the UV and the X-ray peak luminosity (Hawley et al., 1995; MitraKraev et al., 2005). They estimated a proton flux associated with the flare of $5.9 \times 10^{8}$ protons $\mathrm{cm}^{-2} \mathrm{sr}^{-1} \mathrm{~s}^{-1}$ for particles with energies $>10 \mathrm{MeV}$. Then they calculated the abundance of nitrogen oxides produced by the flare by scaling the production of these compounds during a large solar proton event called the Carrington event (e.g., Cliver and Dietrich, 2013). 
Results of Segura et al. (2010) indicate that the UV radiation emitted during the flare does not produce a significant change in the ozone column depth of the planet. When SEPs were included, the ozone depletion reached a maximum of $94 \% 2$ years after the flare for a planet with no magnetic field. At the peak of the flare, the surface UV flux in the wavelength ranges that are damaging for life exceeded those received on Earth for a duration of less than $100 \mathrm{~s}$. The atmospheric column then recovered and reequilibrated within $\sim 50$ years. Segura et al. (2010) concluded that flares may not present a direct hazard for life on the surface of an orbiting habitable planet.

Recent studies have been performed to determine the effect of flare electromagnetic energy on the habitability of planetary systems. The TRAPPIST-1 system has been investigated by Vida et al. (2017) in a recent Kepler/K2 study showing at least $\sim 0.75$ cumulative flares per day of energy between $1.26 \times 10^{30}$ and $1.24 \times 10^{33} \mathrm{erg}$. This could produce detrimental UV flux at the potentially habitable planetary surfaces of TRAPPIST-1b-h. O'Malley-James and Kaltenegger (2017) investigated the potential UV-related surface habitability of the TRAPPIST-1 system and found that the oxic state of the atmosphere is key to protecting the surface, with even a thin-oxygen atmosphere ( $\sim 0.1$ bar $)$ sufficient to keep UVC from reaching the surface with any intensity. Estrela and Valio (2017) have identified superflares in the Kepler-96 system up to $\sim 1.8 \times 10^{35} \mathrm{erg}$, simulated the effects on both Archean and present-day Earth-like atmospheres, and found that the presence of an $\mathrm{O}_{3}$ layer is crucial to protection of life under such highly irradiating events.

Aside from Segura et al. (2010), these previous studies do not investigate the effects of proton events associated with stellar magnetic activity, and they do not study the resilience and evolution of the $\mathrm{O}_{3}$ column of a potentially habitable planet to such events. AD Leonis, the star used for the study by Segura et al. (2010), is one of the most magnetically active M dwarfs known. In the past few decades, only flares from the most active $M$ dwarfs were studied because the UV emission from mid- and low-active red dwarfs falls below the detection threshold of the available instruments. Observations performed using the Hubble Space Telescope showed that UV emission from chromospheric activity was also present on those stars usually classified as non-active (Walkowicz et al., 2008; France et al., 2012, 2013), while the Kepler/K2 mission (e.g., Davenport et al., 2014; Hawley et al., 2014) and the MOST instruments (Davenport et al., 2016) showed a more detailed view of flare frequency from low-mass stars.

The present work extends the results from Segura et al. (2010) to determine the effect of multiple $\mathbf{M}$ dwarf flares and energetic proton events on a potentially habitable world by taking advantage of the more recent observational campaigns noted above. We have updated the models used in Segura et al. (2010) (see Section 2.1) to study the effect of multiple events on an Earth-like atmosphere to determine the potential effects on the $\mathrm{O}_{3}$ column and related biologically relevant UV flux at the surface of the planet.

\section{Methods}

\subsection{Improvements extending Segura et al. (2010)}

A modified version of the $1 \mathrm{D}$ coupled radiative-convective and photochemical model used by Segura et al. (2010) is used in the present work. The radiative-convective model is a hybrid of two models: a $\delta$ two-stream scattering model for reflected stellar radiation, and a more rapid radiative transfer model (RRTM) for thermal wavelengths. The $\delta$ two-stream scattering algorithm solves coupled differential equations to calculate radiative fluxes in the downward and upward directions, through plane-parallel, vertical slices of atmosphere (e.g., Toon et al., 1989); atmospheric absorption in this model uses the method of four-term, correlated- $k$ coefficients which allows for broadened Voigt profiles at specific altitudes in the atmosphere over 38 spectral intervals for important atmospheric species, for example, $\mathrm{O}_{3}, \mathrm{CO}_{2}, \mathrm{H}_{2} \mathrm{O}$, and $\mathrm{CH}_{4}$ (e.g., Kasting and Ackerman, 1986). For thermal-IR wavelengths, an independent RRTM implemented by Segura et al. (2003) was used. The RRTM uses 16-term sums in each spectral band where $k$-coefficients concentrated where the most rapidly changing radiative conditions are occurring, that is, allowing for improved spectral resolution where Doppler broadening is important.

The photochemical model solves 217 reactions that link 55 chemical species. Photolysis was calculated using a $\delta$ two-stream routine that allowed scattering between molecular gases and sulfate aerosol species (Segura et al., 2003). Time steps were solved using an implicit reverse Euler method, with initial time step set to $10^{-4} \mathrm{~s}$, with increasing magnitude as the system reaches equilibrium.

The coupling layer allowed the climate and photochemical model to cross-communicate and synchronize the atmospheric temperature structure, $\mathrm{H}_{2} \mathrm{O}$ profiles, and chemical alterations made for each time step. The pressure layers calculated in the radiative-convective model were interpolated to the fixed altitude structure in the photochemical model, and then back, during the coupling procedure. All other details and inputs to the model not discussed below are identical to those discussed in Sections 2-3 in Segura et al. (2010).

In the present work, we made four important modifications: Section 2.2 describes how we unfixed the mixing ratios for three major atmospheric constituents $\left(\mathrm{N}_{2}, \mathrm{O}_{2}\right.$, and $\mathrm{CO}_{2}$ ) to achieve a more realistic mass balance; Section 2.3 details the treatment used for multiple flare events and flare light curve evolution based on recent observations; Section 2.4 discusses the considerations made regarding the interaction between multiple stellar proton events and the upper atmosphere; Section 2.5 discusses the extension of the stellar optical-NIR spectrum during flare events.

\subsection{Unfixed mixing ratios for $\mathrm{O}_{2}, \mathrm{CO}_{2}, \mathrm{~N}_{2}$ and Henry coefficients}

For the single-flare work addressed in Segura et al. (2010), the volume mixing ratios, $f$, of major atmospheric constituents $\mathrm{O}_{2}, \mathrm{CO}_{2}$, and $\mathrm{N}_{2}$ were fixed at values corresponding to $0.21,3.55 \times 10^{-4}$, and 0.78 , respectively. In the present work, these mixing ratios are enforced only at the surface layer of the planet, and all layers of the atmosphere above the surface were unfixed-allowing these species to photochemically respond to the stellar inputs.

Of particular note are the responses of $\mathrm{O}, \mathrm{O}_{2}$, and $\mathrm{N}_{2}$ during the creation of $\mathrm{NO}_{x}$ species generated by proton events. Previous studies have found that each ion pair (positive/negative species) created in the upper atmosphere from precipitating proton ionization events results in the 
production of $1.27 \mathrm{~N}$ atoms, particularly $\mathrm{N}(4 \mathrm{~S})$ and $\mathrm{N}(2 \mathrm{D})$ (Porter et al., 1976), and a $\mathrm{NO}_{x}$ production rate of 1.3-1.6 per pair (Rusch et al., 1981). The generation of $\mathrm{NO}_{x}$ is driven by the dissociation of $\mathrm{N}_{2}$ into the constituent, excited $\mathrm{N}$-atoms, which then react with $\mathrm{O}_{2}$ to produce $\mathrm{NO}$ and $\mathrm{O}$ :

$$
\begin{aligned}
& \mathrm{N}(4 \mathrm{~S})+\mathrm{O}_{2} \rightarrow \mathrm{NO}+\mathrm{O} \\
& \mathrm{N}(2 \mathrm{D})+\mathrm{O}_{2} \rightarrow \mathrm{NO}+\mathrm{O}
\end{aligned}
$$

and $\mathrm{O}_{3}$ reacts with the produced $\mathrm{NO}$ to generate $\mathrm{NO}_{2}$ :

$$
\mathrm{NO}+\mathrm{O}_{3} \rightarrow \mathrm{NO}_{2}+\mathrm{O}_{2}
$$

Following the method of proton injection in Segura et al. (2010), we directly modify the atmospheric column to simultaneously include the $\mathrm{NO}$ and $\mathrm{NO}_{2}$ profiles at or near the peak of the flare events; the excited $\mathrm{N}$ atoms resulting from proton precipitation and ion production are not directly injected. $\mathrm{NO}_{x}$ species are included in the model at steady state, from the start at $t=0$, and are then scaled throughout the atmospheric profile from $20-65 \mathrm{~km}$ at or near the flare peak according to the proton fluence for a flare of a given energy, and dependent on the altitude. The same altitude profile for $\mathrm{NO}_{x}$ injection is used for each event, only the magnitude of injected $\mathrm{NO}_{x}$ profile is reliant upon flare energy. An important limitation of this approach is that we cannot obtain information regarding differences in the atmospheric $\mathrm{NO}_{x}$ or $\mathrm{O}_{3}$ columns from repeated flaring as a function of proton event energy spectra. In the present work, as well as that of Segura et al. (2010), the $\mathrm{NO}_{x}$ scaling profile is related to the northern hemisphere proton spectrum for March 23, 1991, reported by Rodger et al. (2008). An event with a harder or softer proton spectrum will produce different atmospheric chemistry due to, for example, ionization rates, increased or decreased precipitation altitude, and so on. More discussion on proton event scaling is below in Section 2.4.

The amount of $\mathrm{NO}_{x}$ directly input into the upper atmosphere to emulate production by proton precipitation (and therefore pair production) sets the amounts of $\mathrm{N}_{2}$ and $\mathrm{O}_{2}$ removed by the formulating reactions (as well as $\mathrm{O}_{3}$, and the addition of $\mathrm{O}$ ). For each $\mathrm{NO}$ molecule we inject, $0.5 \mathrm{~N}_{2}$ and $1 \mathrm{O}_{2}$ molecules were immediately removed from the atmospheric column, and one $\mathrm{O}$ atom was added. For the case of $\mathrm{NO}_{2}$ molecules injected at a specific altitude, the abundances are predicate on the previous formation of $\mathrm{NO}$, as seen in Eq. R3; during the formation of $\mathrm{NO}_{2}$ from $\mathrm{NO}, 1 \mathrm{O}_{3}$ molecule is removed, and per the reactant $\mathrm{NO}$ that drove the production of the $\mathrm{NO}_{2}, 0.5 \mathrm{~N}_{2}$ molecules are removed from the atmospheric column, and $1 \mathrm{O}$ atom is added. Note that the product $\mathrm{O}_{2}$ from Eq. R3 is cancelled by the assumed preproduction of the $\mathrm{NO}$ molecule, which consumes an $\mathrm{O}_{2}$ as in Eqs. R1 and R2. For simulations containing multiple proton events, we limit the maximum $\mathrm{NO}\left(\mathrm{NO}_{2}\right)$ injected into the atmosphere by the amount of available reactant $\mathrm{O}_{2}$ $\left(\mathrm{O}_{3}\right)$ in the atmospheric column.

As a consequence of unfixing the mixing ratio of $\mathrm{CO}_{2}$, its abundance was increased in the stratosphere by methane oxidation (Yung and DeMore, 1999), as we will show in Section 4.3.

Rainout and saturation for the new unfixed species were also modified to be temperature dependent, and the appro-
Table 1. Henry Coefficients, Mixing Ratio at Surface

\begin{tabular}{lccc}
\hline Species & $\begin{array}{c}\mathrm{k}_{H} \\
\left(\text { mol kg }^{-1} \text { bar }^{-1}\right)\end{array}$ & $\begin{array}{c}d\left(\ln \left(\mathrm{k}_{H}\right)\right) / d(\mathrm{l} / \mathrm{T}) \\
(K)\end{array}$ & $\begin{array}{c}\mathrm{f}(\mathrm{z}=0) \\
(\text { arb })\end{array}$ \\
\hline $\mathrm{O}_{2}$ & $1.3 \times 10^{-3}$ & 1500 & 0.21 \\
$\mathrm{CO}_{2}$ & $3.55 \times 10^{-2}$ & 2400 & $3.55 \times 10^{-4}$ \\
$\mathrm{~N}_{2}$ & $6.25 \times 10^{-4}$ & 1300 & 0.78 \\
\hline
\end{tabular}

Henry coefficients and surface mixing ratios for each unfixed species.

priate Henry coefficients are shown in Table 1 along with the initial mixing ratios at the surface level of the atmospheric column. The model uses the parameterization proposed by Giorgi and Chameides (1985) to calculate the removal of the soluble species. Each long-lived species is assigned a solubility using Henry's law (for most of them) or an "effective" solubility for those species that dissociate in solution $\left(\mathrm{SO}_{2}\right.$, for example). For this case, the $\mathrm{pH}$ of the raindrops is calculated at each layer of the troposphere of the model by taking into account the relevant gaseous and dissolved species, typically $\mathrm{CO}_{2}, \mathrm{SO}_{2}$, and $\mathrm{H}_{2} \mathrm{CO}$, using a Newton-Raphson step to solve a system of 10 coupled algebraic equations. After the raindrop $\mathrm{pH}$ is calculated, effective solubilities are obtained, and the method of Giorgi and Chameides (1985) is applied to calculate rainout rates. The parameterization uses a proportionality constant that depends on the water profile and species solubility and includes the episodic nature of the wet removal process.

All of the above changes to the 1D model did not significantly alter $\mathrm{O}_{2}$ and $\mathrm{N}_{2}$ during steady state from their uniform altitude mixing ratio profiles of 0.21 and 0.78 , respectively.

\subsection{Multiple flare events from Kepler observations}

To address the effects of multiple flares on the atmosphere, we built upon the AD Leo flare template used in the previous work of Segura et al. (2010) by using statistical results from Kepler observations of the M4 star GJ1243. We incorporated the observations of the flare energies, durations, and amplitudes from GJ1243 (Hawley et al., 2014), which included analysis of over 6100 GJ1243 flares measured over 11 months at 1 min cadence. These observations also resulted in the empirical flare light curve evolution template we employ (Davenport et al., 2014). The following are scaling relations derived from Hawley et al. (2014) that we used to determine flare amplitude (relative increase in U-band flux), flare frequency, and event durations:

$$
\begin{gathered}
\log _{10} \frac{\Delta F_{\mathrm{U}}}{F_{\mathrm{U}}}=0.761 \log _{10} E-25.86 \\
\log _{10} \nu=-1.01 \log _{10} E+31.65 \\
\log _{10} t=0.395 \log _{10} E-9.269
\end{gathered}
$$

where $F_{\mathrm{U}}$ is the stellar flux in the Johnson U-band, $E$ represents the flare energy in ergs, $v$ is the flare frequency given in cumulative flares per day, and $t$ is the duration of the flare events in seconds, which ranges from $\sim 600 \mathrm{~s}$ to $\sim 14,500 \mathrm{~s}$ 


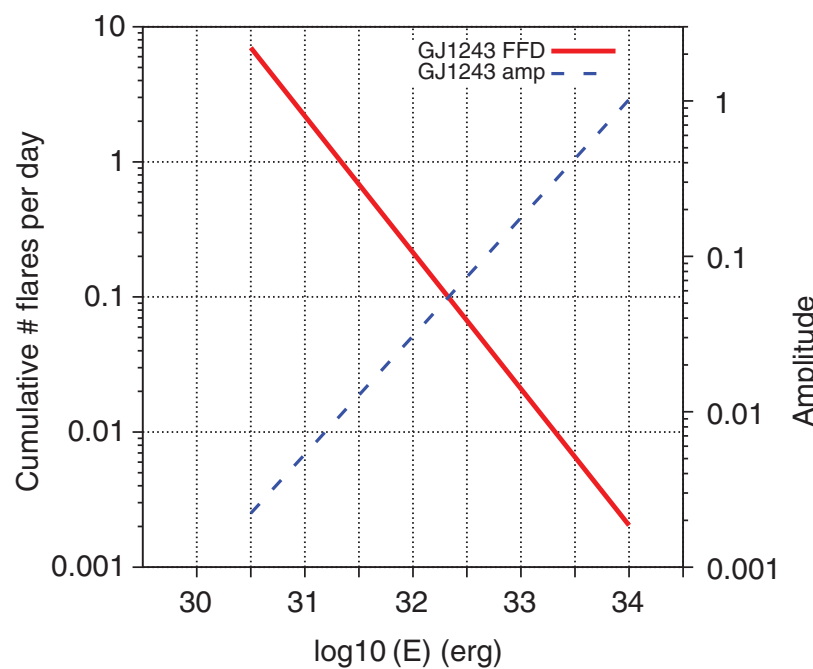

FIG. 1. The FFD and amplitudes observed from GJ1243 used to generate flare distributions in the present work.

for flares of energy $10^{30.5} \mathrm{erg}$ and $10^{34} \mathrm{erg}$, respectively. Figure 1 shows a plot of the flare frequency and amplitudes as a function of the energy used to generate our flare distributions.

The present work explores several separately generated distributions of stellar flare events with duration of 1 month, 6 months, 1 year, 10 years, and 15 years. We simulated flares at a rate of $\sim 7$ per day with energies in the Johnson U-band from a minimum of $10^{30.5} \mathrm{erg}$ up to a maximum of $10^{34} \mathrm{erg}$, which occurs with frequency of 1 flare per $\sim 489$ days according to the flare frequency distribution (FFD) for GJ1243 reported in Hawley et al. (2014) and shown in Fig. 1. An example extended, temporal flare distribution with $\sim 1277$ (2555) flares over 6 months ( 1 year) was generated from the GJ1243 FFD and can be seen in Fig. 2. As seen in Fig. 1, a relative flare amplitude of $\sim 1.03$ corresponds to a flare with energy $10^{34} \mathrm{erg}$ - approximately the energy of the great flare of AD Leo reported by Hawley and Pettersen (1991) —and $\sim 2.24 \times 10^{-3}$ corresponds to a flare of size $10^{30.5} \mathrm{erg}$ from GJ1243.

Cliver and Dietrich (2013) estimate the solar Carrington event of 1859 to be on the order of $5 \times 10^{32} \mathrm{erg}$, events which by Eq. 1 have a relative amplitude of $\sim 0.106$. However, any reference to "Carrington-sized" flares in the present work were calibrated by proton fluence $\left(\sim 1.1 \times 10^{10} \mathrm{~cm}^{-2}\right)$, rather than total flare energy, and correspond to a total energy of $\sim 10^{31.9}$ $\mathrm{erg}$, with a flux amplitude of $\sim 0.026$. Another event of reference that was likely impactful to Earth's atmosphere was the large SEP event of $775 \mathrm{AD}$ evident in geological records (e.g., Mekhaldi et al., 2015; Sukhodolov et al., 2017), which is estimated to have had proton fluence of $\sim 4 \times 10^{10} \mathrm{~cm}^{-2}\left(E_{\mathrm{p}} \geq\right.$ $30 \mathrm{MeV}$ ). This fluence corresponds to a total flare energy of $\sim 10^{32.75} \mathrm{erg}$, or relative flux increase of $\sim 0.116$ in the present work (see the scaling discussion below in Section 2.4).

For all events, flare flux was stacked additively, enabling the simulation of complex flaring events as seen in the inset of the top panel in Fig. 2. The additive stacking of flare flux represents an upper limit of particle flux for proton events.

The flare light curve template was also modified in the present work. Following Davenport et al. (2014), we con-
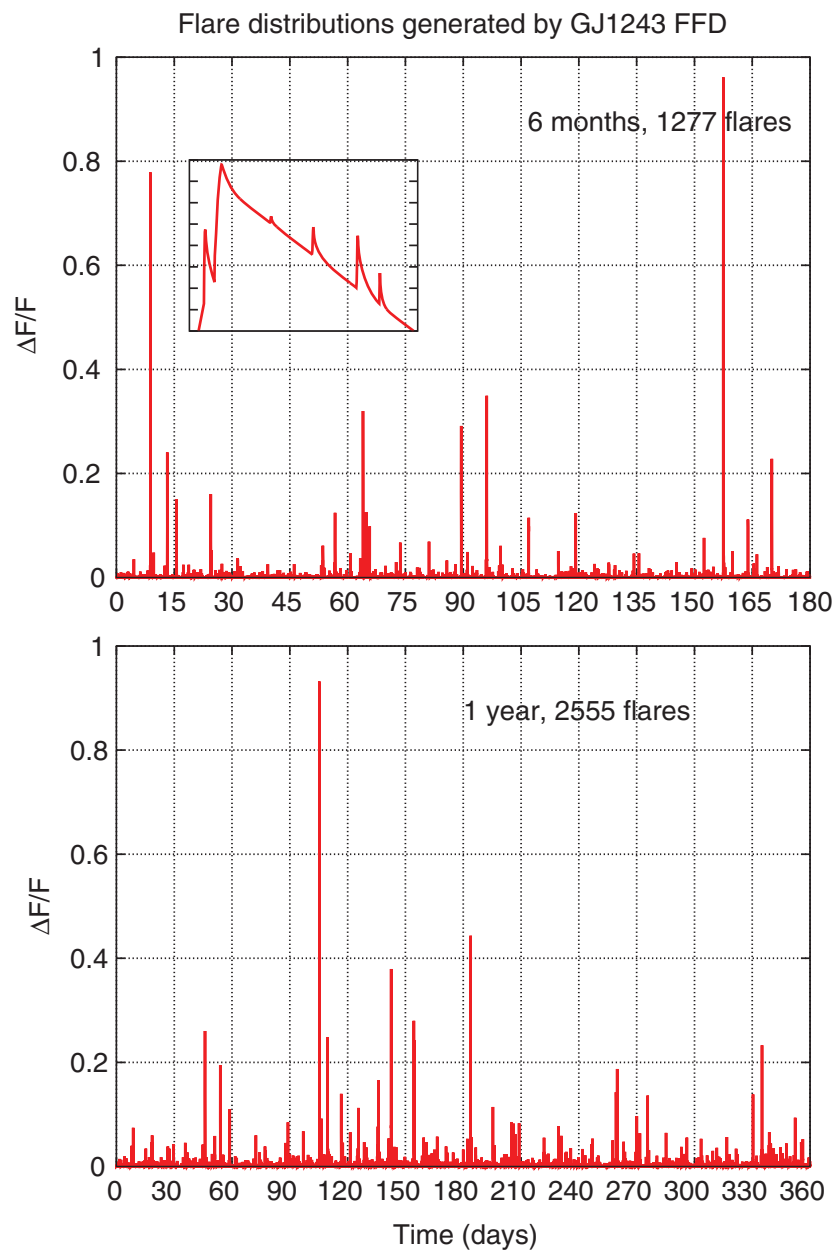

FIG. 2. Top: Six months, flare distribution (1277 events) generated from the GJ1243 FFD. The inset identifies an example complex flare near $\sim 9$ days, produced by flare flux stacking. Bottom: One-year flare distribution (2555 events) from the GJ1243 FFD.

structed each flare event using a light curve template granting a rapid impulsive phase and extended decay phase. Figure 3 shows a relative comparison between the light curve shape used in Segura et al. (2010) and an equivalent $10^{34} \mathrm{erg}$ flare from the present work for the first $6000 \mathrm{~s}$ of the flare events. It is worth noting that the difference between peak times results mostly from how one defines where a flare starts; with the template applied, we start from a much smaller increase in magnitude for the first time step compared to Segura et al. (2010).

Integrating the impulsive phase from the start of the event to the peak for each flare results in a factor of $\sim 0.71$ in flux delivered for the treatment in the present work (solid blue) from Davenport et al. (2014), compared to integrated results from the Segura et al. (2010) light curve (dashed red). Integrating each flare from start to end, however, results in the present treatment depositing approximately twice the overall flux when compared to the treatment in Segura et al. (2010). Integrating both curves across the full-width, half-maximum (FWHM) results in a factor of $\sim 1.72$ increase of the flux compared to the original treatment in Segura et al. (2010). In the present work, the majority of the flare energy is 


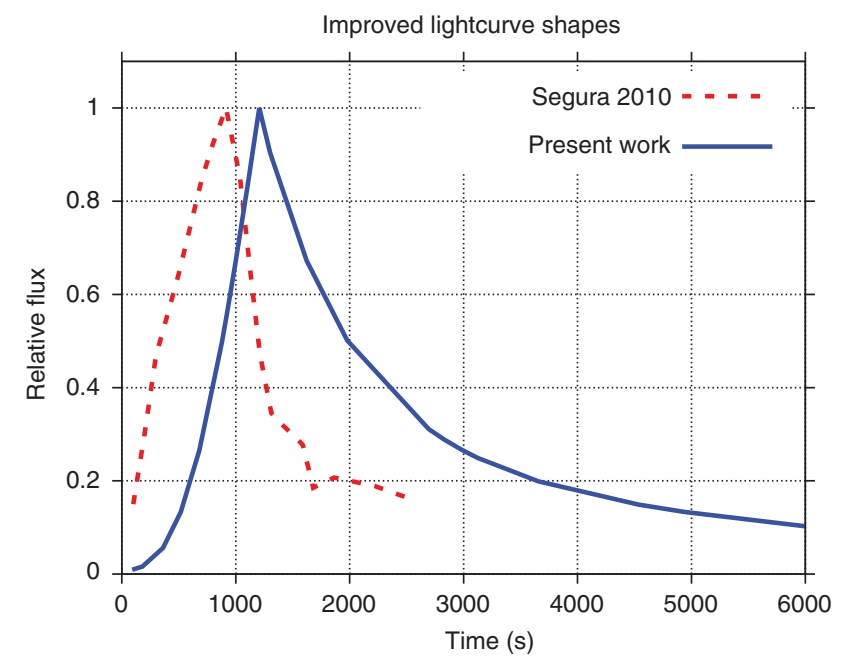

FIG. 3. Comparison of light curve evolution from Segura et al. (2010) vs. the method adopted in the present work, based on the empirical modeling results of Davenport et al. (2014).

delivered in the decay of the flare evolution due to the long timescale compared to the impulsive phase. It is worth noting that for flare energies $<10^{34} \mathrm{erg}$, the FWHM, rise and decay times (and therefore total duration, as seen in Eq. 3) all occur at reduced scale with the decreased energies.

The per-flare time-step resolution was increased in our implementation to be more granular, from 20 flare points used in the 2010 work to 30 points (12 in the rise phase instead of 9, 18 in the decay phase instead of 11) to obtain proper coverage of temporal flare morphology. The resulting flare in Fig. 3 is $5.6 \times$ the duration of the flare used in Segura et al. (2010) ( $\sim 14,500 \mathrm{~s}$ versus $\sim 2,586 \mathrm{~s})$ due to the long decay phase from the empirical template adopted from Davenport et al. (2014). This duration varies with the flare energy according to Eq. 3.

\subsection{Proton event scaling and impact probability}

In addition to UV radiation, flare events can produce proton events that can drive changes in atmospheric chemistry. In this section, we explain our process and reasoning behind the scaling of proton events for the flare energy ranges simulated, and the likelihood of their impact on an orbiting planet. Essentially, we follow the scaling method of Segura et al. (2010), relying on a correlation between flare $\mathrm{X}$-ray flux and proton flux. Our method produces results that match well with observations at Earth during the well-studied March 1991 flare event noted in the modeling of Rodger et al. (2008). We use this particular 1991 proton event in the present work to scale $\mathrm{NO}_{x}$, expanding the method to the orbital and stellar parameters for our extrasolar system of interest. We should note that the use of this particular spectrum to scale $\mathrm{NO}_{x}$ for each proton event in the present work is idealized for the purposes of modeling, since real spectral distributions are not constant with increasing flare energy over 4 orders of magnitude. Additionally, there is uncertainty of proton event spectral characteristics for $\mathrm{M}$ dwarfs, as particle energization at a highly active, fully convective star could differ from the solar magnetic environment.
Scaling X-ray intensity is used to estimate proton flux from flare events. We assume the spectral X-ray energy density in the 1-8 $\AA$ bandpass derived in Segura et al. (2010) for the $10^{34}$ erg flare in Hawley and Pettersen (1991) scales with our relative flare flux amplitude relation from Eq. 1. The relationship we employ between peak X-ray intensity and proton flux is given by Belov et al. (2005):

$$
I_{\mathrm{p}}(>10 \mathrm{MeV})=4.8 \times 10^{7} I_{x}^{1.14}
$$

This relationship relative to our overall flare flux (or flare energy) is shown in Fig. 4. The relationship between relative flare flux and proton flux units is expressed in Eq. 5:

$$
I_{\mathrm{p}}(>10 \mathrm{MeV})=k_{0}\left(\frac{\phi^{1.08}}{a^{2}}\right)^{1.14}
$$

where $k_{0}$ is a constant with a value of $\sim 9.0603 \times 10^{6}$, $\phi=\Delta F / F$ is the relative flux increase in the $\mathrm{U}$ band from Eq. 1 , and $a$ is the planetary orbital semimajor axis in astronomical units (in this work, $a=0.16 \mathrm{AU}$ ).

Belov et al. (2005) showed a correlation between X-ray intensity and proton flux for solar events observed over three solar cycles, using the IMP-8 and GOES satellite instruments. The GOES satellites orbit at geosynchronous altitudes, implying the GOES measurements of proton flux are typically taken from within Earth's magnetosphere, except during strong flow due to a fast solar wind stream or the impact of a coronal mass ejection (CME). By this reasoning, the scaling relation we use here from Segura et al. (2010) and Belov et al. (2005) is based upon the magnetized Earth applied to our unmagnetized extrasolar planet, so it can therefore be assumed to be a lower bound of proton fluence for the latter.

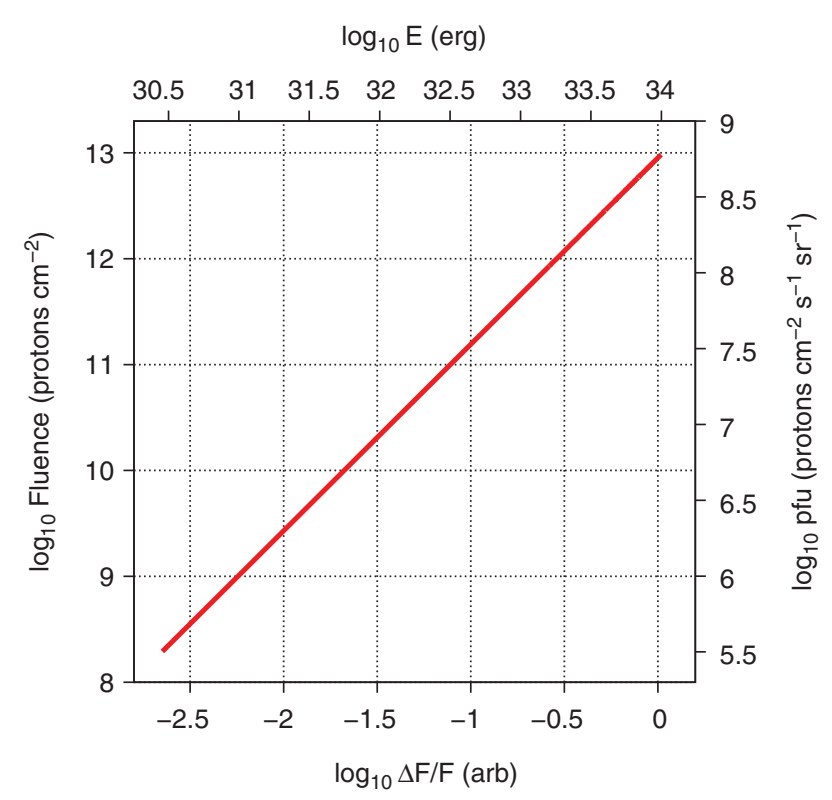

FIG. 4. The relationship of proton fluence (left $y$ axis) and proton flux units (pfu, right $y$ axis) for the flares simulated in the present work, as function of relative flux increase (bottom $x$ axis) and total flare energy (top $x$ axis). 
To ensure our scaling is reasonable, we apply it and compare to the March 1991 observed solar event at Earth. Keeping in mind the variability for solar and magnetospheric dynamics, we compare here our proton event flux scaling with the well-studied solar proton event of the March 22/23, 1991, flare that is classified as a GOES X9.4 event, that is, peak X-ray flux $9.4 \times 10^{-4} \mathrm{~W} \mathrm{~m}^{-2}$ in the $1-8 \AA$ bandpass as measured by the GOES-6/7 instruments. The GOES 6 and 7 satellites measured peak (average, $4 \mathrm{~h}$ window centered on peak) proton fluxes of $\sim 20,900(\sim 10,900)$ and $\sim 43,500$ $(\sim 22,500)$ proton flux units, respectively (pfu, $\phi$ in Eq. 5 above, in particles $\mathrm{cm}^{-2} \mathrm{~s}^{-1} \mathrm{sr}^{-1}$ ), for protons with energy $\geq 10 \mathrm{MeV}$ during an event that had a duration of $\sim 19.5 \mathrm{~h}$. At the time of peak proton flux unit measurement, the GOES satellites were well inside Earth's magnetosphere.

We follow the scaling used in Segura et al. (2010) based on Belov et al. (2005), which is noted above in Eq. 5, and find that a GOES X9.4 flare (with relative flux, $\phi$, of $\sim 2.049 \times 10^{-4}$ by our scaling) would produce $17,003 \mathrm{pfu}$ at Earth, which seems reasonable when compared to the average GOES-6/7 measurements above and a bit underscaled from the peaks (at most by a factor of two). For our purposes, we are assuming all of our calculated flux reaches the upper atmosphere of the planet; for this single, energetic solar event, at least, the scaling matches well. To be sure, we must keep in mind that we are making many assumptions (e.g., $\mathrm{M}$ dwarf $\mathrm{X}$-ray/proton scaling is similar to solar scaling, absence of Earth-like geomagnetic field). It is worth noting that due to deflection by the planetary magnetic field at Earth, protons with energies of less than $\sim 50 \mathrm{MeV}$ would not easily penetrate to the GOES instrumentation at geostationary orbit $\left(6.6 R_{\mathrm{E}}\right)$, so the measured values are not representative of the potential proton fluxes measured outside the magnetosphere-which may be higher.

Here, we discuss the energy ranges and expected proton fluences for the present work's simulated flares. For the low end of our $\mathrm{M}$ dwarf flare energy scale, the $10^{30.5} \mathrm{erg}$ flare gives a $\geq 10 \mathrm{MeV}$ proton flux of $\sim 3.23 \times 10^{5} \mathrm{pfu}$ (fluence of $\sim 1.93 \times 10^{8}$ protons $\mathrm{cm}^{-2}$ ), corresponding to a $1-8 \AA$ bandpass flux of $1.25 \times 10^{-2} \mathrm{~W} \mathrm{~m}^{-2}$ from our scaling method or a GOES X124 equivalent solar flare. The top-end energy of $10^{34} \mathrm{erg}$ scales to $\sim 6.13 \times 10^{8} \mathrm{pfu}$ (fluence of $\sim 9.46 \times 10^{12}$ protons $\mathrm{cm}^{-2}$ ), corresponding to $9.34 \mathrm{~W} \mathrm{~m}^{-2}$ in the $1-8 \AA$ bandpass (approximately the same flux calculated in Segura et al. [2010]), or a GOES X93,400 solar flare. This is certainly a regime with much uncertainty, given no such events have ever been recorded for the Sun.

One of the largest recent solar events, measured by the STEREO-A spacecraft, occurred on the backside of the Sun in July 2012 (Russell et al., 2013; Gopalswamy et al., 2016). The proton spectrum of this event was hard, even compared to the spectrum of the event modeled in our work from March 1991, with fluence that rivaled estimates for the Carrington event, $10^{9}$ to $10^{10}$ protons $\mathrm{cm}^{-2}$ for $E_{\mathrm{p}}>10 \mathrm{MeV}$, and an estimated proton flux of up to $\sim 64,000$, based on the measured proton spectra (Gopalswamy et al., 2016).

Not all flares necessarily produce energetic proton events. However, with the above analysis in mind, we use the following reasoning to assume all stellar flare events modeled in the present work have a proton event associated. It has been reported that $100 \%$ of solar flare events of class GOES $\mathrm{X} 2$ or higher have energetic proton events (e.g., CMEs,
SEPs) associated with them (e.g., Yashiro et al., 2006; Hudson, 2011; Dierckxsens et al., 2015). That corresponds to an X-ray flux of $2 \times 10^{-4} \mathrm{~W} \mathrm{~m}^{2}$ over the $1-8 \AA$ bandpass. As mentioned above, following the assumptions made in Segura et al. (2010), the lowest-energy flare in the present work would be roughly equivalent to a GOES X125 flare from the Sun-larger than any recorded event. Using this as proxy for the $\mathrm{M}$ dwarf events, it is not unrealistic to assume a one-to-one flare-CME frequency correlation for our work.

Since not all CMEs are guaranteed to be directed toward an orbiting planet, we want to take into account the geometries of emitted energetic proton events, (e.g., Khodachenko et al., 2007; Kay et al., 2016). Khodachenko et al. (2007) calculates the probability of a planet being hit by a flare-related CME as

$$
P_{\mathrm{CME}}=\frac{\left(\Delta_{\mathrm{CME}}+\delta_{\mathrm{pl}}\right) \sin \left[\left(\Delta_{\mathrm{CME}}+\delta_{\mathrm{pl}}\right) / 2\right]}{2 \pi \sin \Theta}
$$

where $\Delta_{\mathrm{CME}}$ is the angular size of the CME, $\delta_{\mathrm{pl}}$ is the solid angle subtended by the planet, and $\Theta$ is the hemispheric latitude range of CME activity on the star. Here, we study two sets of values related to common solar observations of $\Delta_{\mathrm{CME}}=5 \pi / 18(\pi / 2)$ as a restrictive (permissive) estimate, and stellar magnetic latitude $\Theta= \pm \pi / 4$ in both cases; $\delta_{\mathrm{pl}} \approx$ $10^{-8}$ is negligible for an Earth-like planet at $0.16 \mathrm{AU}$; further, we assume zero orbital inclination. We also assume that CME activity expands similarly to solar geometry, instead of being trapped in the astrospheric current sheet. This gives a probability for every CME to hit our simulated planet of $P_{\mathrm{CME}}=0.083(0.25)$ for the restrictive (permissive) CME angular size. In this work, we assume all particles in our calculated fluence impact and precipitate into the atmosphere of the planet, ignoring potential deflection from a planetary magnetic field. In Section 4.7, we discuss the potential complications and suggest directions for future work.

\subsection{Optical-red flare continuum}

In Segura et al. (2010), the flare spectrum used to drive the photochemistry included flux from the far UV to 4500 A. During the 20 time steps in the flare, flux from wavelengths greater than $4500 \AA$ was not scaled to flare levels from the blackbody continuum associated with quiescence. In the present work, we extended all flare time steps to include increased flux out to the near IR, to $8500 \AA$.

To use a more realistic spectrum, the optical-red continuum wavelengths longward of $4500 \AA$ for each time step were scaled to the relative flux value at $4500 \AA$ and then extended to $8500 \AA$ by adding the modeled combined tails of two blackbody continuum curves with maximum temperatures of 5,000 and $10,000 \mathrm{~K}$ to represent the increased optical-red spectrum from the flare (the so-called "conundrum") following Kowalski et al. (2013). Figure 5 shows the extended flare spectra during the impulsive, rising phase of the flare from 2000 to $8500 \AA$, with our extended, upscaled spectrum to the right of the vertical dashed red line at $4500 \AA$. The time steps in the decay phase of the flare events were similarly scaled.

\section{Results}

The following subsections demonstrate the resulting effects of repeated stellar flares, with and without CME/SEP 
FIG. 5. Full UV-visible spectrum for the impulsive phase of a characteristic AD Leo great flare-sized $\left(10^{34} \mathrm{erg}\right)$ event. The spectra to the right of the vertical dashed red line have been added in the present work.

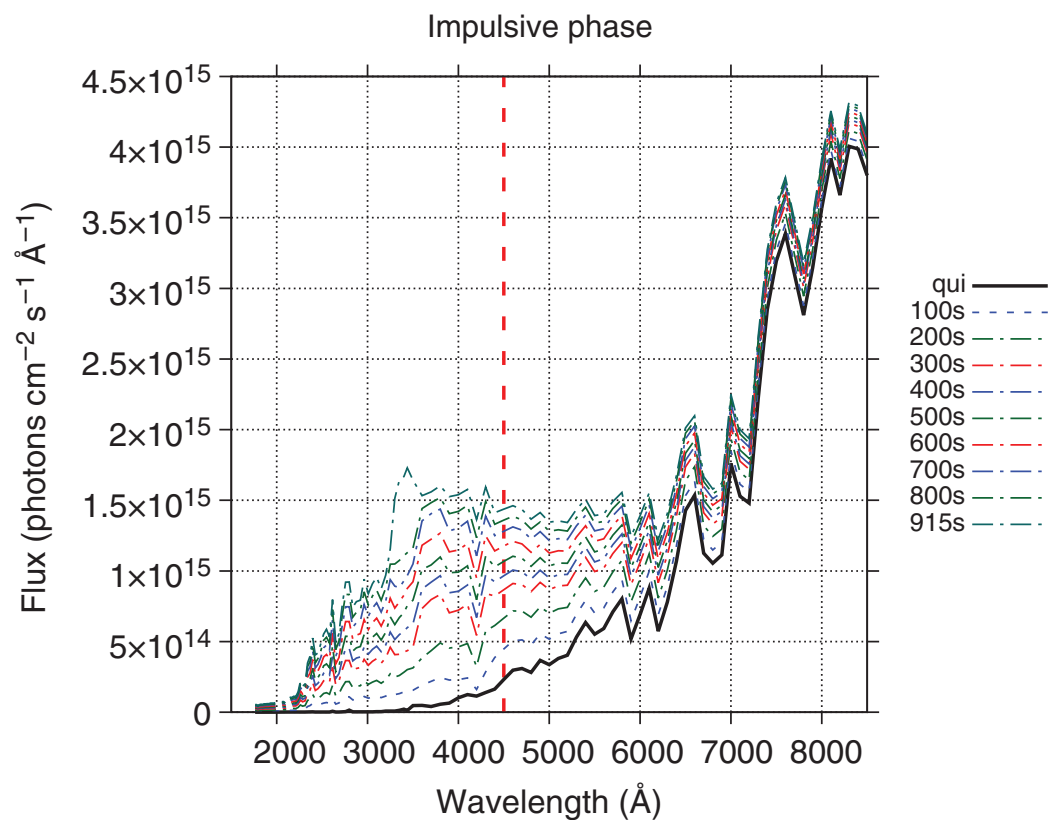

events, on the atmosphere and surface UV flux of an unmagnetized Earth-like planet at 0.16 AU.

The 1D photochemical model runs for each time step throughout every simulation. For results in Sections 3.1 (energy parameter-space comparison) and 3.2 (temporal parameter-space comparison), the time steps during the flare are set by the energy scaling characteristics and light curve timing from GJ1243 (e.g., Davenport et al., 2014; Hawley et al., 2014), discussed above in Section 2.3, and vary from order $10^{0}$ to $10^{3} \mathrm{~s}$. For Section 3.3 (EM-only FFD generated events) and 3.4 (EM+proton FFD generated events), the entire flaring period for all durations was run with a $1 \mathrm{~m}$ cadence; the coupled 1D radiative-convective model is run between flares, typically for intervals of order $\geq 10^{3} \mathrm{~s}$ both during interflare periods, and the postflare recovery phase is run with a variable time step, that is, the method used in
Segura et al. (2010). It is worthwhile to note here that due to the high frequency of flare activity, the atmosphere does not typically return to an equilibrated steady state between flare events, as would be the case for a planet orbiting such an active stellar host. The timescale to equilibrium for individual flare events can be seen in Fig. 6 .

\subsection{Energy parameter-space comparison} with Segura et al. (2010)

We simulated the atmospheric effects of an array of single-flare energies from the range covered by the GJ1243 FFD and compared the results to the original AD Leo-sized flare $\left(10^{34} \mathrm{erg}\right)$ simulated in Segura et al. (2010). The effects of electromagnetic-only (EM-only) and electromagnetic with proton events (EM+protons) were both simulated; the

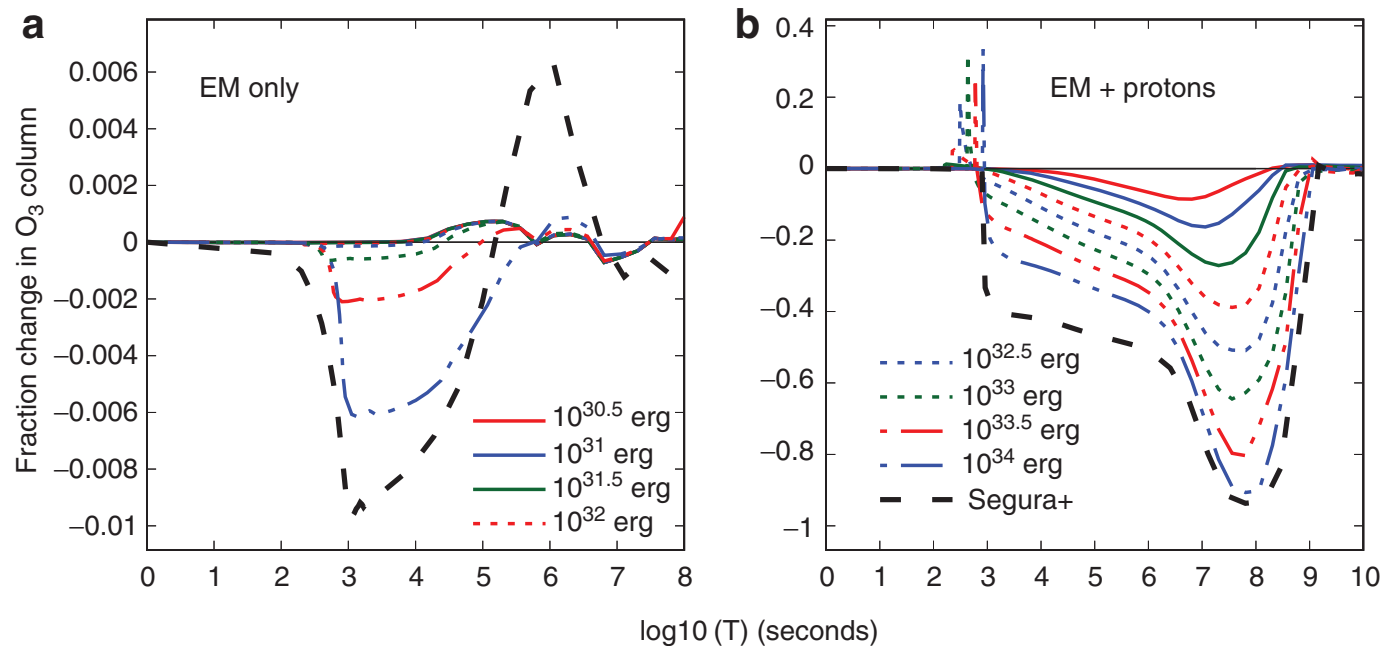

FIG. 6. $\mathrm{O}_{3}$ evolution for single-flare energy parameter comparison with Segura et al. (2010) for EM-only (a) and $\mathrm{EM}+$ protons (b) $10^{30.5}$ to $10^{34}$ erg flares. 
proton flux and fluence for each flare (see Fig. 4) are correlated with the amplitude of the flare as given by the Kepler observational results as described in Section 2.3, Eq. 1, and as discussed above in Section 2.4.

Figure 6 shows the single-flare effects on the ozone column for EM-only and EM+protons in the left and right panels, respectively, as a function of time. The $10^{34} \mathrm{erg}$ EMonly case in Fig. 6a is a direct comparison of the new model (dash-dotted blue line, with updates included from Section 2.1) to the original flare simulated in Segura et al. (2010) (dashed black line). In the new treatment, the magnitude of $\mathrm{O}_{3}$ column depletion is reduced, and the depressed column density recovers more gradually due to the long decay phase of the flare event. Applying the flare template from Davenport et al. (2014) to the flare spectral evolution discussed in Section 2.3 (e.g., new treatment has wider FWHM, less impulsive, longer decay phase) is indicated in the overall impact on the $\mathrm{O}_{3}$ column as seen in these single events. The results suggest that EM-only flare impulsivity has a moderate impact on the $\mathrm{O}_{3}$ column, when compared to the overall flux delivered to the atmosphere, whereas the atmospheric recovery time is more dependent on the flare duration or overall energy.

For EM-only lower-energy flare events $\left(\leq 10^{32} \mathrm{erg}\right)$, the overall effect is to initially decrease the $\mathrm{O}_{3}$ column by a small amount (factor of $10^{-4}$ ) and subsequently increase the ozone column by a fractional percentage $(<1 \%)$. At lower flare energy, the flare duration is fairly short (e.g., $600 \mathrm{~s}$ for $10^{30.5} \mathrm{erg}$ ). The generation of ozone via free $\mathrm{O}$ atoms from photodissociation of other species $\left(e . g ., \mathrm{H}_{2} \mathrm{O}\right)$ occurs more rapidly than the direct photodissociation of $\mathrm{O}_{3}$, initially resulting in the overall slight increase of the $\mathrm{O}_{3}$ column. Once the energy of the modeled EM-only flares becomes sufficiently large $\left(>\sim 10^{32} \mathrm{erg}\right)$, the overall $\mathrm{O}_{3}$ photodissociation rates are significant enough to produce a notable net loss of ozone due to the flux input from a single flare event. The long-term effects of this slight increase in $\mathrm{O}_{3}$ column depth by these low-energy, high-frequency EM-only events are discussed and shown below in Section 3.3.

Figure $6 \mathrm{~b}$ shows the EM+proton cases, including a direct comparison of the new model (colored lines) with the results of Segura et al. (2010) (dashed black line). The most significant difference featured in runs from the present work is the rapid increase and subsequent decrease in the ozone column at the time of $\mathrm{NO}_{x}$ injection, and is driven by the mass-balancing addition of $\mathrm{O}$ at an equivalent rate to the $\mathrm{NO}$ injected as discussed in Section 2.2. In these events, $\mathrm{NO}_{x}$ is injected at the peak of the flare, as in Segura et al. (2010), the timing of which increases with increasing overall flare energy, due to the related increase in both flare rise/decay times relative to flare durations (e.g., Hawley et al., 2014), as noted in Eq. 3. The immediate subsequent decrease of the $\mathrm{O}_{3}$ generated by the injected $\mathrm{O}$ atoms is seen, for example, due to the rapid action of $\mathrm{NO}_{x}$ destruction of $\mathrm{O}_{3}$.

The integrated $\mathrm{NO}_{x}$ scaled to the total energy of the proton event is injected at the peak of the flare. This approach is taken because we do not use a specific shape for the evolution of the proton events, and the reactions that produce $\mathrm{N}$ and $\mathrm{NO}_{x}$ are faster (Ejzak et al., 2007) than the flare time steps in our model, and so would be largely completed by the end of the flare.

Comparing the two $10^{34} \mathrm{erg}$ flares in Fig. 6b (present work = blue dash dotted line, Segura et al. [2010]= black da- shed line), we find a difference between the two events, with a peak ozone loss of $\sim 90 \%$ (94\%) occurring $\sim 2$ years after the event for the present work (Segura et al., 2010), and recovery taking $\sim 50$ years in both cases. It is apparent that events with the highest impact on the atmosphere are those including stimulated $\mathrm{NO}_{x}$-production through energetic proton flux into the upper atmosphere, as is evidenced by comparing the lowest $\mathrm{EM}+$ proton event at $10^{30.5} \mathrm{erg}$, which shows a factor of $\sim 14$ greater $\mathrm{O}_{3}$ column loss $(\sim 8.4 \%)$ when compared to the highest EM-only $10^{34}$ erg flare in the left panel $(0.6 \%)$. The proton flux, and therefore $\mathrm{NO}_{x}$ production, for all $\mathrm{EM}+$ proton events was scaled according to the method outlined in Section 2.4 and seen in Fig. 4. This corresponds to a total fluence for the $10^{30.5}$ erg event of $\sim 1.95 \times 10^{8}$ protons $\mathrm{cm}^{-2}$, or a factor of $2.03 \times 10^{-5}$ of the simulated AD Leo event, and a factor of $\sim 1.33 \times 10^{-2}$ of the Carrington event of 1859 (Rodger et al., 2008). Taking into account this scaling for lower-energy flare events, the ozone column is still significantly altered, with peak ozone column loss of $\sim 8.4 \%$ occurring $\sim 2.5$ months after the event; for our simulation of a Carringtonsized proton event, peak $\mathrm{O}_{3}$ loss of $\sim 36 \%$ equilibrium value occurs at $\sim 1.15$ years after the flare.

Repeated impacts by proton events even at the lowest energies considered could be particularly destructive to the ozone column of the Earth-like planet, depending on orbital parameters, as well as CME frequency and geometries. The resulting effect of multiple proton events on the $\mathrm{O}_{3}$ column and surface UV flux is discussed below in Sections 3.2, 3.4, and 4.2.

\subsection{Temporal spacing of flare activity, with and without protons}

We simulated the effect of multiple flares with varying temporal spacing to parameterize the effect of flare frequency on atmospheric evolution, notably the $\mathrm{O}_{3}$ column. Two sets of simulations were performed, EM-only and EM+proton events. The EM-only simulations were performed with 1000 flares, over five different cases that set the interflare separation periods at $2 \mathrm{~h}, 1$ day, 1 week, 1 month, and 1 year. Note that the spacing between flare events is measured from the final time step of the initial flare event and the first time step of the following flare event. From Fig. 6a, one can see that single lower-energy flares do not significantly impact the ozone column, so the results shown in Fig. 7 to determine the effect of EM-only flare spacing were all performed with the AD Leo-like $10^{34} \mathrm{erg}$ flare flux to generate the worst-case effect of UV on the ozone column. In Section 3.3, the effects of the lower-energy EMonly events can be seen during the simulations generated from the GJ1243 FFD. Simple pairs of flare events were also simulated for both EM-only and EM+protons but showed little deviation from single events and are not shown here.

Figure 7 shows the results for the EM-only simulations, consisting solely of multiple $10^{34}$ erg flares. The results indicate that the electromagnetic events from active $\mathbf{M}$ dwarfs impact an atmosphere slowly, but perhaps over long enough time-significantly. The case with a 1-year interflare separation (dashed blue line) shows no appreciable change over the course of $\sim 1000$ years, as the period of atmospheric recovery for a single $10^{34}$ erg event is $\sim 1$ year (as seen in Fig. 6a); therefore, each flare is affecting a nearly re- 
FIG. 7. The effects on the $\mathrm{O}_{3}$ column of EM-only, $10^{34} \mathrm{erg}$ flares with varying interflare separations. Separations of 1 day, 1 week, 1 month, and 1 year included simulations of $10^{3}$ flares, where the $2 \mathrm{~h}$ separation included $10^{4}$ flares to obtain extended effects for long-term prediction of $\mathrm{O}_{3}$ column (dash-dotted red line).

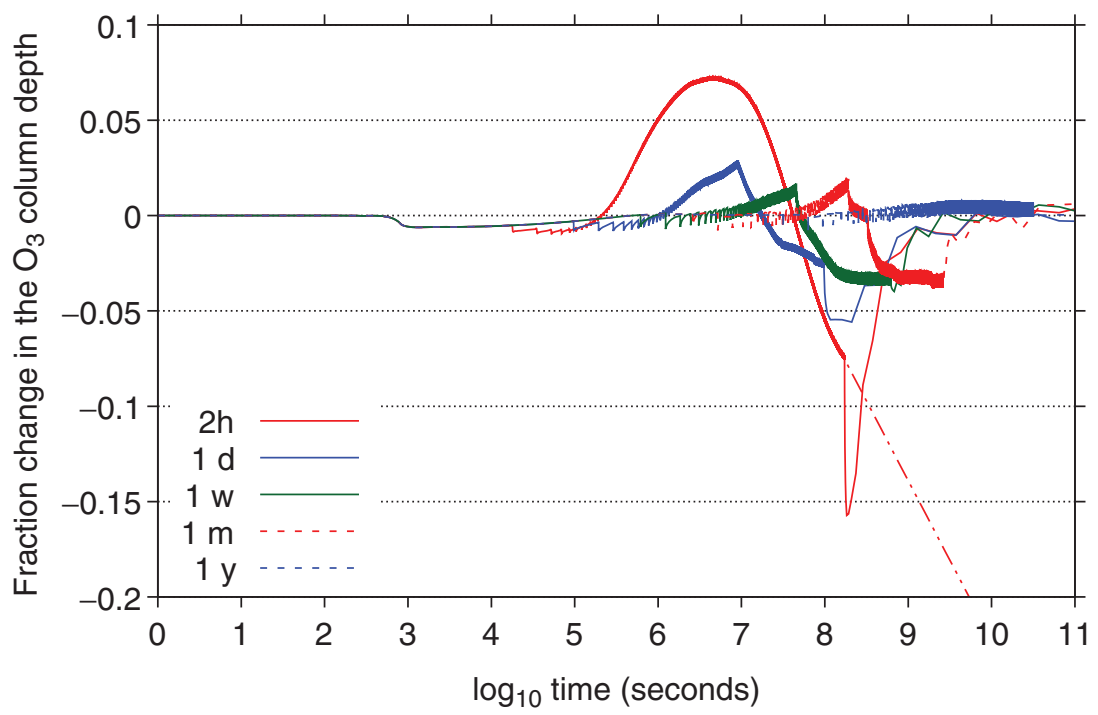

equilibrated atmosphere, leading to seemingly small change over periods of time of order 1000 years. It should be noted here that the active star used in the flare model-the AD Leo spectra applied to the atmosphere via the GJ1243 FFD and light curve-would experience a flare of this magnitude approximately once every 489 days, as seen in the stellar FFD in Fig. 1.

The cases run with interflare separation of 1 day, 1 week, and 1 month all suggest that extended periods of frequent events equivalent to the great flare of AD Leo slowly erode the $\mathrm{O}_{3}$ column over extended periods but seem to reach a potential new equilibrium at $\sim 97 \%$ of the steady state value. This gives rise to some concern for a potentially habitable planet, as $\mathrm{M}$ dwarfs stay active for well into their multi-billion-year lifetimes on the main sequence (e.g., Silvestri et al., 2005). However, instead of focusing on the loss rate for those flaring frequencies, we will comment on the simulation with $2 \mathrm{~h}$ interflare separation: the worst-case scenario from a star much more active than AD Leo or GJ1243. In this case, $10^{4}$ flares were run instead of $10^{3}$, which corresponds to a period of $\sim 6.9$ years. At that point, the $\mathrm{O}_{3}$ column has been eroded by only $\sim 7.4 \%$ of the equilibrium value, albeit at a very steep rate of decline.

To estimate the continued loss rate if this high amount of flux continually impacts the planetary atmosphere, we extend the dot-dashed red trendline in Fig. 7 and find that even by the age of the current universe $\left(\sim 4.3 \times 10^{17}\right.$ s) the $\mathrm{O}_{3}$ column loss would only be $\approx 86.1 \%$. This is less impactful than the full impact of a single proton event from a $10^{34} \mathrm{erg}$ flare. This result is discussed primarily as an extreme thought experiment, as such conditions are highly unlikely to persist for a significant portion of planetary evolution: no $\mathrm{M}$ dwarf has been observed experiencing 12 AD Leo great flare-sized events per day. It is possible that very young stars could exhibit such activity, though, so these results could be applied to very early evolution of an Earth-like atmosphere containing significant oxygen content (e.g., Luger and Barnes, 2015; Meadows, 2017; Meadows et al. 2018b).

The EM+proton simulations were performed with 100 flares, with each event producing proton fluence that impacts the habitable zone planet at the same interflare sepa- ration periods mentioned above. In reality, CME and SEP events could be glancing blows, or miss the planet entirely, as discussed in Section 2.4. We chose to simulate energies of $10^{30.5} \mathrm{erg}$, the lowest energy included in this work; $10^{31.9}$ erg events with approximately equivalent proton fluence to the Carrington event; and $10^{34} \mathrm{erg}$, equivalent to the $\mathrm{AD}$ Leo flare. In Fig. 8, we show the results from 100 proton events for these three different energies. The black dashed line in each panel shows the impact of a single proton event of each energy for reference.

In general, the results suggest that even the lowestenergy, most frequent flares from a star like GJ1243-10 30.5 erg-can rapidly erode the $\mathrm{O}_{3}$ column of an Earth-like planet for all interflare frequencies except the once-per-year case. Table 2 shows the time to destroy $\geq 99 \%$ of the $\mathrm{O}_{3}$ column for the cases in the middle (Carrington-like, $10^{31.9}$ erg) and bottom (AD Leo-like, $10^{34}$ erg) panels in Fig. 8. To predict these time-to-loss values, we used a log fit to the $\mathrm{O}_{3}$ column depth curve for the last $\approx 1000$ points during the period of active flaring and then extrapolated to find the intercept at $99 \%$; the method assumes that the loss continues at the rate of the last 1000 or so points of $\mathrm{O}_{3}$ column evolution during flare activity. Note that assuming the loss rate is maintained is possibly inaccurate, as one can see from the cases simulated for 10-year duration in Fig. 11 and discussed in Section 3.4-the response at long times tends to follow a separate fit, which declines more gradually. For this reason, we do not extrapolate from the $10^{30.5}$ erg results in the top panel of Fig. 8; instead, we discuss the impact of these lower-energy flares below, in Section 3.4.

If every flare from a star as active as GJ1243 hits a planet, we might expect the rate of $\mathrm{O}_{3}$ depletion to be quite rapid, similar to what is seen in the top panel of Fig. 8, for the $2 \mathrm{~h}$, 1-day, and 1-week interflare separations. One can see that for the 1-year interflare separation, the trend flattens out, and the atmosphere reaches a new steady state at $\sim 86 \%$ of the original steady state column depth. Similarly, for the 1month interflare simulations, the trend begins a flattening just before the end of the flare activity, likely reaching a new equilibrium around $50 \%$ of steady state. 


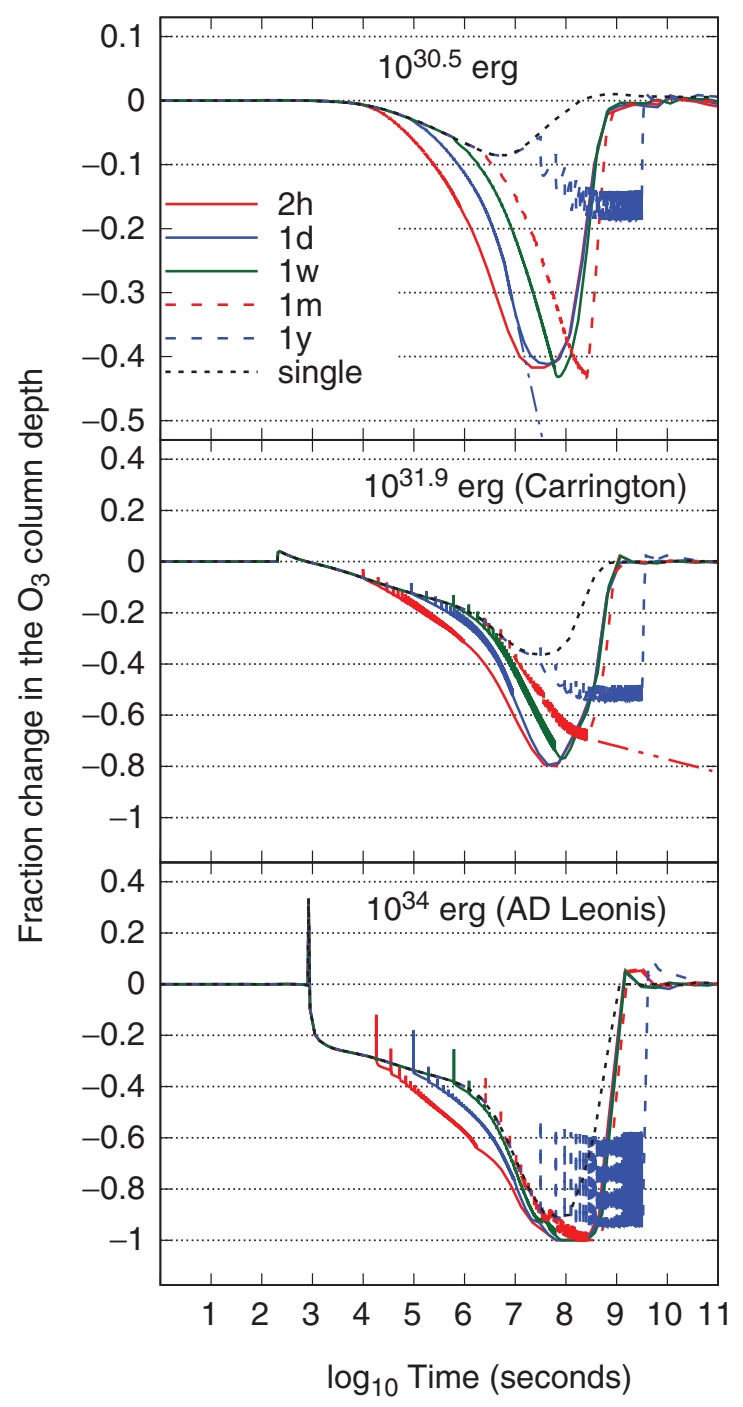

FIG. 8. $\mathrm{O}_{3}$ evolution driven by repeated proton events, 100 flare events for all cases. Top: $10^{30.5} \mathrm{erg}$ flares+protons. The $1 \mathrm{~d}^{-1}$ period is likely to occur for a planet orbiting GJ1243; the dash-dotted blue line extrapolates the predicted $\mathrm{O}_{3}$ loss rate. Middle: Carrington equivalent proton events at $10^{31.9}$ erg. The dash-dotted red line predicts $\mathrm{O}_{3}$ evolution for the most likely frequency experienced at a GJ1243-orbiting planet. Bottom: AD Leo equivalent proton events with $10^{34} \mathrm{erg}$.

Taking into account the CME/SEP geometries discussed in Section 2.4, along with the FFD, the most likely case for a planet orbiting a star like GJ1243 to experience a Carringtonlike event is in bold in Table 2. For the Carrington-like $10^{31.9}$ erg flares, one flare occurs every $\sim 3.7$ days. Assuming the CME probability of 0.08 , this implies one proton event hits the planet every $\sim 46$ days. In this case, applying the power law fit suggests an $\mathrm{O}_{3}$ lifetime of $\sim 2.64 \times 10^{14} \mathrm{~s}$, or $\sim 8.4$ Myr for a planet's $\mathrm{O}_{3}$ column to be effectively destroyed. Note that prolonged simulations may show a change in the trend as the atmosphere is able to recover from multiple flaring events such as these-see discussion in Section 3.4. However, note that under continuous, repeated flaring, the atmosphere is unlikely to recover. As shown in longer-term simulations involving the impacts of supernovae proton
Table 2. Time Estimation in Seconds to $\geq 99 \% \mathrm{O}_{3}$ LOSS, BY INTERFLARE SEPARATION

\begin{tabular}{lcc}
\hline & $10^{31.9}$ erg & $10^{34.0}$ erg \\
\hline 2 hour sep & $3.50 \times 10^{7}$ & $1.89 \times 10^{7}$ \\
1 day sep & $7.38 \times 10^{7}$ & $3.22 \times 10^{7}$ \\
1 week sep & $2.55 \times 10^{8}$ & $8.33 \times 10^{7}$ \\
1 month sep & $\mathbf{2 . 6 4 \times \mathbf { 1 0 } ^ { 1 4 }}$ & $1.35 \times 10^{8 *}$ \\
Total fluence $\left(\right.$ prot $\left.\mathrm{cm}^{-2}\right)$ & $1.82 \times 10^{12}$ & $8.49 \times 10^{14}$ \\
\hline
\end{tabular}

Time (in seconds) to $\geq 99 \% \mathrm{O}_{3}$ loss assuming continued flare activity beyond the 100 flares simulated as shown in Fig. 8. Power law fits to $\mathrm{O}_{3}$ loss rate for last $\sim 20$ flares in each case, and are shown for the most likely cases for a planet orbiting GJ1243. Time in bold represents the most likely time to loss for a planet orbiting GJ1243 in the habitable zone for conservative CME/SEP geometries (see Section 2.4).

*For the $10^{34} \mathrm{erg}$ flares with 1 -month interflare separation, the system sustained $\mathrm{O}_{3}$ losses $\geq 99 \%$ during the flaring period at this time above, no fit necessary.

cosmic rays (much higher particle energies and flux than are simulated here), the likely best-case scenario is likely that the $\mathrm{O}_{3}$ column will asymptotically approach and equilibrate at a new, reduced level (e.g., Melott et al., 2017; Thomas, 2017). Note that similar asymptotic equilibration under repeated particle injection events is the behavior suggested by results here, and in the following sections.

In the case of the multiple $10^{34}$ erg flares, one can see in the bottom panel of Fig. 8 and in Table 2 that each interflare frequency simulated has the potential to reduce the $\mathrm{O}_{3}$ column depth to $1 \%$ of its original value in less than 3 years for all but two cases. The $2 \mathrm{~h}$ separation case should reach that level of $\mathrm{O}_{3}$ destruction more rapidly, though here it was cut off after 100 flares, or $\sim 200 \mathrm{~h}$. Proton events at this energy impacting the planet with this once-per-two-hour frequency are unlikely for a host like GJ1243, but a more active M dwarf or very active early star could produce such events. The other outlier is the lowest frequency, with one flare per year. A host like GJ1243 would experience one $10^{34}$ erg flare once every $\sim 489$ days, so the once-per-year case (blue dashed line) is a rough approximation to the expected atmospheric response if each of the events hit the planet. This case indicates that the $\mathrm{O}_{3}$ column is roughly equilibrated at $\sim 94 \%$ loss, with the oscillations representing the short-term increase in $\mathrm{O}_{3}$ at the point of initial $\mathrm{NO}_{x}$ injection discussed in Section 2.2. However, as the discussion in Section 2.4 notes, approximately one in eight (or one in four) proton events of all energies hits the planet, depending on CME geometry.

For all of these EM+proton simulations, the results in Table 2 assume that only these proton events are impacting the planetary atmosphere, ignoring other less and more energetic proton events ejected by the stellar host as would be seen in a realistic flare distribution such as Fig. 2. Note that for complex flaring events, where the flux is stacked as seen in the inset in the top panel of Fig. 2, an average flux of particles is represented, as opposed to a sum of the proton flux. The simulations including realistic flare distributions and proton event geometries are shown and discussed below in Section 3.4.

\subsection{EM-only FFD-generated flares}

The results of Segura et al. (2010) and those in Section 3.1 above indicate that single, EM-only flares do not significantly 
impact the atmosphere of the ozone column of an Earth-like planet at 0.16 AU. Results in Section 3.2 suggest that even frequent $10^{34} \mathrm{erg}$ EM-only events are not as impactful as a single, lower-energy proton event. However, given the flaring frequency of active M dwarfs like GJ1243 (Hawley et al., 2014), terrestrial planetary atmospheres for planets orbiting these active hosts will be impacted by multiple flares per day at varying energies. Figure 1 shows the GJ1243 FFD and amplitude for the stellar host's flare activity in our simulations, indicating that $\sim 7$ flares per day of energy $10^{30.5}$ erg and above will impact the planetary atmosphere.

A 6-month (1-year) example distribution of the generated flares is shown in the top (bottom) panel of Fig. 2, showing a generated timeline of 1277 (2555) flares over that 180-day (360-day) period. The flux for each flare was stacked additively, allowing for the effects of simulated complex flares as shown in the log-scale inset for the top panel of Fig. 2; the inset is a zoom around the large flare event of amplitude $\sim 0.78$ ( $\left.\sim 10^{33.84} \mathrm{erg}\right)$ just prior to Day 9. Four such individually generated distributions were created using the GJ1243 FFD and used to drive a more realistic atmospheric simulation response to EM-only flaring for total flaring time of 1 month, 6 months, 1 year, and 15 years. In the 6-month (1-year) simulations shown in Fig. 2, the largest flare is of amplitude $\sim 0.972(0.950)$ or $9.6(9.3) \times 10^{33} \mathrm{erg}$. An average of seven flares occur per day in these distributions, most of low energy, and they are not visible due to the scaling of the figure. The effects on the ozone column of the planet from the EM-only, multiple flaring distributions are shown in Fig. 9.

The simulations for each of the four flaring frequencies in Fig. 9 show that the early ozone evolution is dominated by the multitude of smaller flares. These smaller events increase the overall ozone column steadily and in predictable fashion despite each run being an independent distribution, most easily seen as the varied presence of larger flare events throughout the flaring period, as seen in Fig. 2. The larger flare events $\left(>10^{33} \mathrm{erg}\right)$ can be seen as negative spikes throughout the distributions. All runs reach a peak ozone increase of $8 \%$ around 1 month, and the longer runs then turn back toward equilibrium. The 15-year case runs long enough to drive the ozone column into the loss region, and from the trend for the range of $2-3 \times 10^{8} \mathrm{~s}$, one can extend the rate of change to calculate that this level of maintained loss would result in $\mathrm{O}_{3}$ column loss of $\sim 37 \%$ by the age of the Universe. Compared to the results shown in Fig. 7, the $2 \mathrm{~h}$ interflare period for multiple $10^{34}$ erg flares drove more than twice the $\mathrm{O}_{3}$ depletion in the same period. It is likely that the EM-only flaring can cause a slow change over tens of billions of years, but even small, continuous proton events combined with the EM are significantly more destructive with respect to $\mathrm{O}_{3}$ abundance.

Longer periods still need to be simulated to isolate the longer-term effects of EM-only flaring, and are planned for future work. Given that $\mathrm{M}$ dwarf stars are active for significant portions of their long lives, these results suggest that the ozone column around these stellar hosts is likely to be depleted, quite significantly if proton events are involved, as shown in the next section.

\subsection{EM+proton FFD-generated flares}

To simulate a more realistic planetary atmospheric response to proton events from a stellar host with flare properties similar to GJ1243, we simulated several cases over one year of realistic flare activity generated from the FFD, shown in the bottom panel of Fig. 2. The 10-year simulations were run from a flare distribution (not shown) generated independently from the GJ1243 FFD, and the distribution is separate from the 6-month and 1-year examples shown in Fig. 2. To gauge the effect of CME geometry discussed in Section 2.4 and noted in Eq. 6, we ran two cases-one conservative case, with smaller $\mathrm{CME}$ solid angles $\left(\Delta_{\mathrm{CME}}=\right.$ $5 \pi / 18$ ) which gave each CME an $\sim 8.3 \%$ chance to hit the planet, and one more permissive case with larger CME solid angles $\left(\Delta_{\mathrm{CME}}=\pi / 2\right)$, giving each $\mathrm{CME}$ a $\sim 25 \%$ chance to hit. In both cases, the maximum stellar latitude of magnetic activity driving proton event ejections was constrained to be between $\Theta= \pm \pi / 4$. With the combination of these two toolsFFD flare distribution from observation of GJ1243 and CME geometry-these results represent a more realistic
FIG. 9. $\mathrm{O}_{3}$ evolution for EM-only flare events generated from the GJ1243 FFD for periods of 1 month, 6 months, 1 year, and 15 years. The dash-dotted black line predicts continued effects of flaring beyond 15 years.

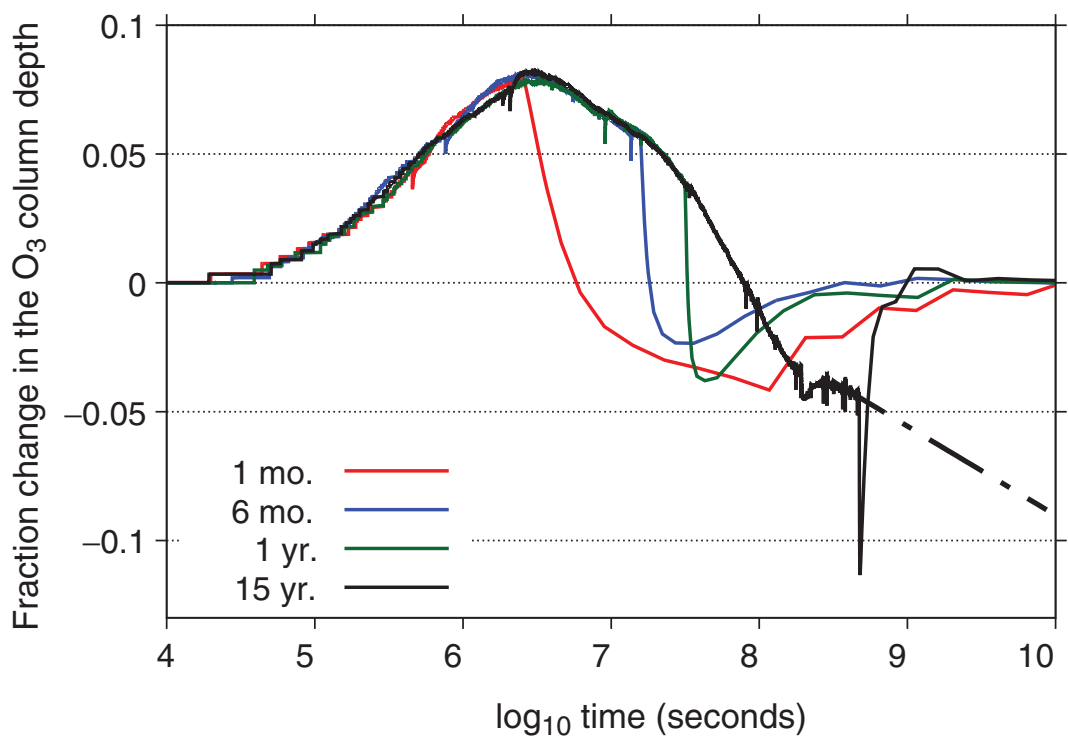




\section{$\log _{10}$ Fluence (protons $\mathrm{cm}^{-2}$ )}

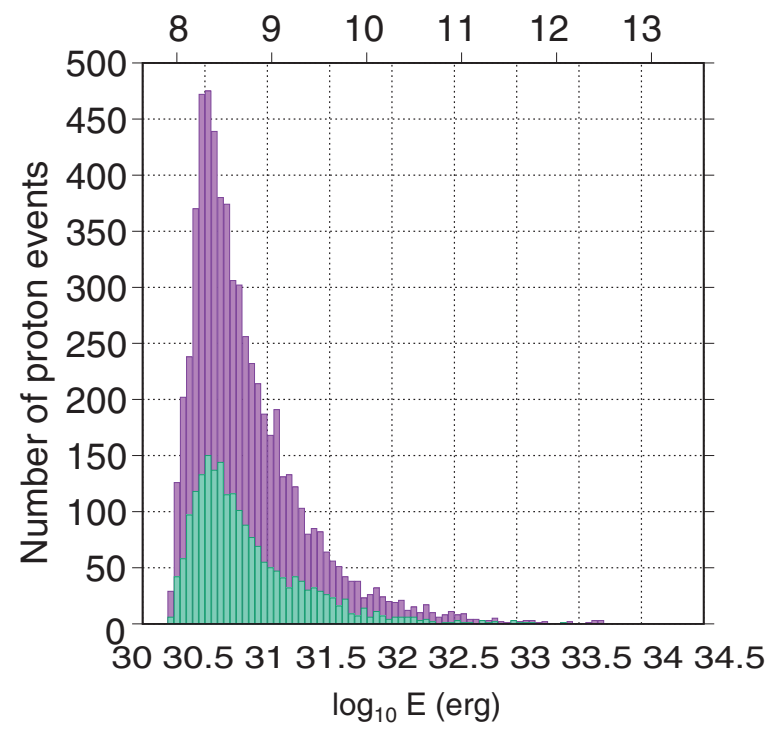

FIG. 10. Distribution of proton events by flare event energy and proton fluence for the 10-year simulations. CME probability $P=0.25(0.08)$ is shown in magenta (green).

representation of the effects of a proton event on the atmospheric evolution for an unmagnetized, Earth-like planet in the habitable zone at $0.16 \mathrm{AU}$ around an active $\mathrm{M}$ dwarf host.

A representative frequency distribution of proton events impacting the planet is shown in Fig. 10; the events were taken from the 10-year duration simulation, the results of which are plotted in Fig. 11 and discussed below. In Fig. 10, the events plotted in green (magenta) represent the CME impact probability of $P_{\mathrm{CME}}=8 \% \quad\left(P_{\mathrm{CME}}=25 \%\right)$, as discussed above. Note there are a few events with less energy and fluence than $10^{30.5}$ erg shown here, as proton events were checked against $P_{\mathrm{CME}}$ and simulated at one of three time steps during each flare event in the simulation: either the peak of the flare or the time step to either side of the flare peak, which allowed the proton fluence to be scaled slightly downward for some events, for example, to $10^{30.2} \mathrm{erg}$ equivalent fluence. The proton events in each case were selected randomly in real-time during each simulation, so the overall distribution of event energies and impact frequencies varies slightly in each of the four 1-year simulations (the randomly selected distribution for the 10-year cases can be seen above in Fig. 10).

The influence on the $\mathrm{O}_{3}$ column is shown in Fig. 11, with the conservative results $\left(\Delta_{\mathrm{CME}}=5 \pi / 18, P_{\mathrm{CME}}=8 \%\right)$ in the top panel and the more permissive results $\left(\Delta_{\mathrm{CME}}=\pi / 2\right.$, $\left.P_{\mathrm{CME}}=25 \%\right)$ in the lower panel. The vertical dashed lines correspond to the end of the flaring periods, that is, 1 and 10 years. The number of impacting proton events in the conservative (more permissive) case in the top (bottom) panel was $199.25 \pm 17.23(595.25 \pm 11.59)$ out of 2555 flares in the 1-year distribution (see bottom panel of Fig. 2), and 2045 (6301) events out of 25,550 impacted the atmosphere of the planet for the 10-year simulations, for $P_{\mathrm{CME}}=8 \%(25 \%)$.

The $\mathrm{O}_{3}$ column responded similarly in each of the four 1year cases (shown as the average = black solid line, and the standard deviation = violet-shaded region), for both restrictive and permissive CME geometries. There, the deviation seen in the 1-year simulations is due to the varying energies from the randomly selected impacting CME events (these can be seen as sudden increase of $\mathrm{O}_{3}$ in each case similar to that discussed in Section 3.1). The more

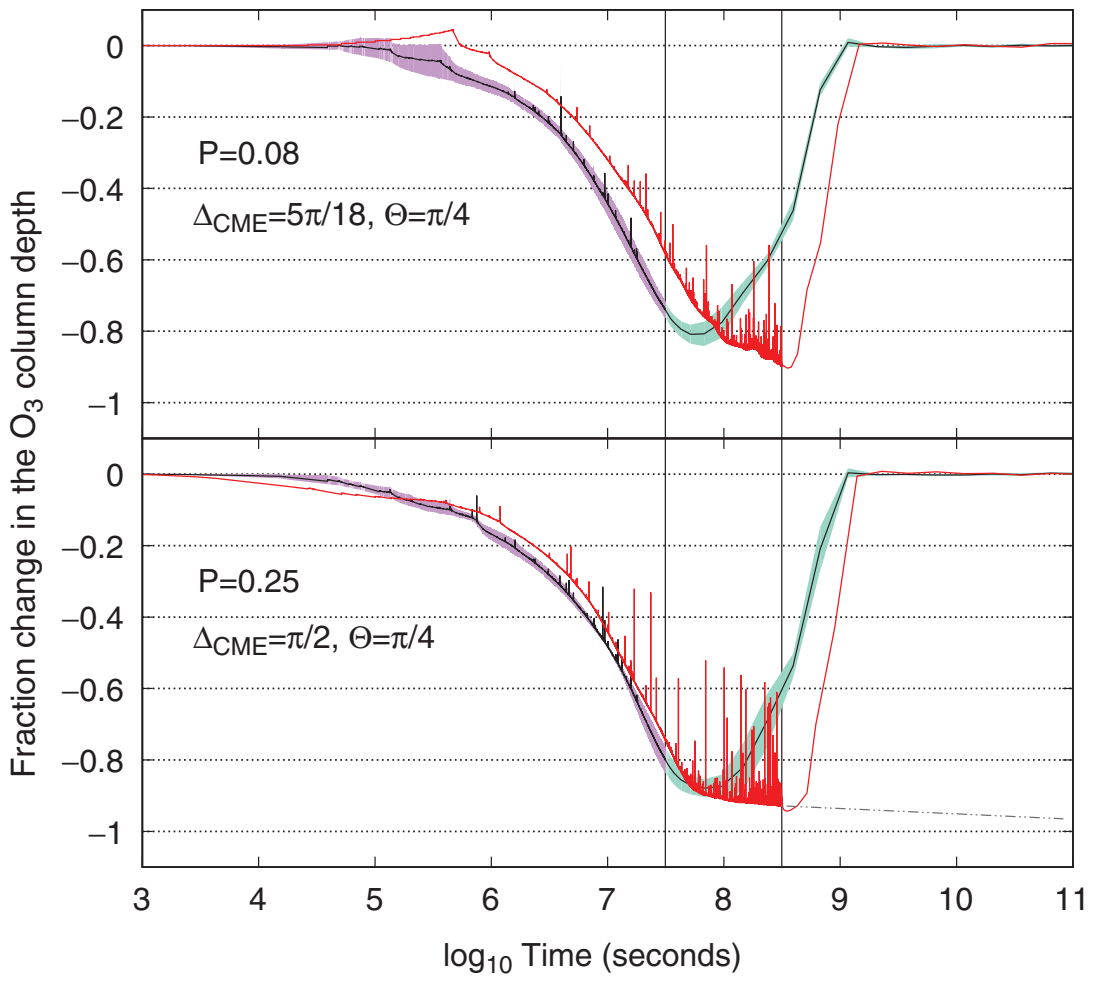

FIG. 11. $\mathrm{O}_{3}$ column depth response to multiple proton events, generated by the GJ1243 FFD and taking into account CME geometries. Vertical lines represent the 1-year and 10-year time steps. The average (black line) and standard deviation for flaring period (shaded violet) and recovery period (shaded green) for 1-year simulations, and one 10-year simulation (red line) are shown. Top: Events with more conservative $\mathrm{CME}$ with per-event probability for impact of $P=0.083$. Bottom: Events with more-permissive geometry with per-event impact probability of 0.25 . 
conservative CME geometry resulted in $\sim 74.10 \pm 2.54 \%$ $\mathrm{O}_{3}$ column loss at the end of the year-long simulations, the first vertical dashed line, and the more permissive geometry resulted in $\sim 80.07 \pm 4.03 \%$ loss.

To determine a more long-term effect, one 10-year run was performed for each CME impact probability, and these are shown in red in both the top and bottom panels of Fig. 11. The 10-year flare distribution was generated separately from the 1-year distribution, and the proton event selection was random and could occur on either the time step of the flare peak or one time step to either side. The $\mathrm{O}_{3}$ column on the last time step for the $P_{\mathrm{CME}}=0.08(0.25) \mathrm{CME}$ impact case is $\sim 10.2 \%(\sim 8 \%)$ of the steady-state equilibrium value. However, note that at the end of these simulations, both cases show a continuing trend of increasing loss. The $P_{\mathrm{CME}}=0.25$ case, in particular, shows a consistent slope that predicts the time to $99 \%(99.9 \%) \mathrm{O}_{3}$ loss as $3.98 \times 10^{12} \mathrm{~s}$, or $126.7 \mathrm{kyr}\left(1.59 \times 10^{13} \mathrm{~s}\right.$, or $\left.502.9 \mathrm{kyr}\right)$, assuming the same loss rate continues. For active, early $\mathrm{M}$ dwarfs, this high level of activity can likely continue for 1$2 \mathrm{Gyr}$, while for later $\mathrm{M}$ dwarfs, that activity lifetime can be 7-8 Gyr (e.g., West et al., 2006, 2008).

It is difficult to simulate the behavior at longer timescales, due to the balance maintained between the time step granularity with which these events need to be simulated for accuracy and the exponential timescales on which these processes occur and could feasibly impact habitability.

\section{Discussion}

All the results in the present work have been performed with a beginning point from an equilibrium Earth-like atmosphere, which by definition assumes an oxygen-rich state. This is worth keeping in mind during the below points of discussion. Future work is planned to investigate varying steady-state conditions for potentially habitable planets in different phases of atmospheric evolution, for example, atmospheres with high $\mathrm{CO}_{2}$, low $\mathrm{O}_{2}$, or haze layers.

\subsection{Multiple events, event frequency, stellar activity}

Below a certain level of proton fluence, the response time of the atmosphere is sufficiently rapid to slow the rate of $\mathrm{O}_{3}$ loss or reach a new equilibrium. This effect can be seen in Fig. 8 as the flattening of the $\mathrm{O}_{3}$ column response toward the later end of the simulations with larger interflare spacing. However, even for smaller flare energies with sufficient frequency - and therefore sustained proton flux - it is possible that $\mathrm{O}_{3}$ recovery is insufficient to prevent a rate of sustained loss to levels $\leq 0.99 \%$ of steady-state equilibrium value.

The results for $10010^{34} \mathrm{erg}$ flare events at a frequency of once per year in the bottom panel of Fig. 8 show the $\mathrm{O}_{3}$ column reaching equilibrium at a loss of $\sim 89 \%$ of the steady-state column depth. This outcome represents the approximate frequency of flaring at this energy level from an FFD like that of GJ1243 (one $10^{34}$ erg event every $\sim 489$ days), and is a loss of UV shielding similar to Segura et al. (2010) (which is important due to flaring with a depressed $\mathrm{O}_{3}$ column as discussed below in Section 4.2), but does not take into account the multiple smaller events, which as discussed in Section 3.4 are very impactful. The results of the more realistic case in Fig. 11, which is based on the FFD of GJ1243, show a non-equilibrium, continual decrease in $\mathrm{O}_{3}$ column depth to $\sim 94 \% \mathrm{O}_{3}$ loss after only 10 years, an order of magnitude smaller time frame than the artificial 100 flares in 100 years above. This implies the continued, rapid deposition of proton events at the low end of the FFD energy range $(\sim 7$ per day for energies $\geq 10^{30.5} \mathrm{erg}$ ) contributes significantly to the depletion of the $\mathrm{O}_{3}$ column, despite a fluence that is less by a factor of $\sim 4 \times 10^{4}$ when comparing the two endpoint values in our adopted FFD $\left(10^{30.5}\right.$ and $\left.10^{34} \mathrm{erg}\right)$; the frequency of the lower-energy events is approximately 3 orders of magnitude higher.

This brings up the issue of whether proton event frequency or fluence dominates the $\mathrm{O}_{3}$ column depletion, which has implications for atmospheric response for planets orbiting smaller, older, or less active stellar hosts. In effect, the average proton fluence delivered over a period of time from GJ1243-level activity is comparable to that of a single moderate flare from the same star. For example, in Fig. 11, the total proton fluence for the 10-year case with more restrictive CME geometry in the top panel was $\sim 9.9 \times 10^{12}$ protons $\mathrm{cm}^{-2}$, roughly equivalent to one $10^{32.7}$ erg flareproduced proton event per year; in the bottom panel, the fluence is roughly equal to one $10^{33.3}$ erg event per year. From Fig. 8, we can interpolate between the Carringtonsized events in the middle panel and the AD Leo-sized events in the bottom panel. Doing so, for fluence from single events per year, the values would equilibrate between roughly $70 \%$ and $75 \%$, not the case we see in Fig. 11, where the 10 -year simulations reach $\mathrm{O}_{3}$ depletion $\geq 90 \%$. For the delivery of proton fluence to the planet, it appears that the frequency of events is as important, if not more important, as the total fluence from the events. Note that it is likely that GJ1243, and other highly active M dwarfs, experience flare events with lower energy and higher frequency than we modeled here but are challenging to measure due to observational constraints.

The total fluence delivered over the $P=0.08(P=0.25)$ single-year simulations in Fig. 11 is $1.49 \pm 0.38 \times 10^{12}$ $\left(4.93 \pm 0.33 \times 10^{13}\right)$. The size of a single Carrington event in the present paper is $1.1 \sim 10^{11}$ protons $\mathrm{cm}^{-2}$ and occurs roughly once per 3.7 days. In comparison, for solar cycles 19-24, the total proton fluence from all measured proton events was of order $10^{10}$ to $10^{11}$ over the 11-12 year periods (e.g., Shea and Smart, 1992; Mewaldt et al., 2005; Lario and Decker, 2011).

Planets orbiting active $\mathrm{M}$ dwarf stars are likely to experience, in a matter of months, massive $\mathrm{O}_{3}$ depletion from proton flux that is multiple orders of magnitude higher than what Earth experiences over entire solar cycles; for planets in the nominal $\mathrm{HZ}$ around these hosts, the particle flux is further increased by a smaller orbital distance compared to Earth, scaling as $1 / R^{2}$, assuming isotropic expansion. Note, however, that the issue is more complicated; isotropic expansion may be a weak approximation due to the turbulent structure of stellar magnetic fields, which contribute to modified radial and longitudinal proton density gradients such that a $1 / R^{2}$ expansion no longer applies. For more active fully convective M3-M5 stars, or active binary systems such as GJ1245AB, one assumes the scaling and complication are even more extreme. 


\subsection{Impact on surface UV flux}

Photodissociating and photoionizing short-wavelength radiation is highly relevant to organic complexity. On one hand, it may be one of the drivers of prebiotic chemistry (e.g., Beckstead et al., 2016; Rapf and Vaida, 2016; Ranjan et al., 2017). On the other hand, UV radiation is responsible for mutations and degradation or transformation of biomolecules which may result in the loss of biological functions (e.g., De Mora et al., 2000); therefore, the shielding effects of an $\mathrm{O}_{3}$ layer for any potentially habitable planet are beneficial to the propagation of organisms on the planetary surface. For example, in Segura et al. (2010), the authors found that the surface UVC flux significantly increased from $<10^{-14}$ up to $10^{-5} \mathrm{~W} \mathrm{~m}^{-2}$ during the peak and recovery phase after an AD Leo-sized proton event. This is 9 orders of magnitude increase over the quiescent value and a UV dose rate for DNA damage of the order of $10 \%$ larger of such a planet.

In the present work, we focus on two aspects: (1) the overall effect of multiple flares on the $\mathrm{O}_{3}$ column-and therefore UV shielding - and (2) the intensity of UV surface flux from flare activity on a planet with an atmosphere already depressed by multiple, prior proton events.

Regarding, point (1) above, we have shown that for stellar hosts with activity such as GJ1243, an $\mathrm{O}_{3}$ column with the starting density of Earth can be reduced by an order of magnitude in only a few years. After this initial rapid loss, the atmosphere seems to move toward a new, lower $\mathrm{O}_{3}$ equilibrium, with the loss rate slowing though not completely at equilibrium. For hosts less active than GJ1243, the situation could be analogous to the top panel of Fig. 8; the atmosphere could reach a new equilibrium in a matter of decades.

Addressing point (2) above, we show surface UV flux results in Fig. 12. The top panel of Fig. 12 represents the results of the $\mathrm{O}_{3}$ depletion shown in both panels of Fig. 11 . The vertical dashed blue lines denote the limits of UVC

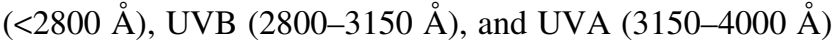
regions. Plots show both quiescent UV flux at the top of the atmosphere (TOA, black dotted) and at the ground (black dash-dotted). The quiescent flux at the ground after the 10year flaring period for CME impact probabilities of $P=0.08$ (dashed black) and $P=0.25$ (dashed red) are also shown. To highlight the upper bounds of UV flux impact, the flux for two flares near the ends of the 10-year runs are shown, at $10^{31.9}$ and $10^{33.6}$ erg for the $P=0.25$ run, with the $\mathrm{O}_{3}$ column at $\sim 8 \%$ of steady-state equilibrium value.

The $\mathrm{O}_{3}$ column at the end of the proton event simulations was $\sim 0.1$ and $\sim 0.08$ of the equilibrium values (as discussed above in Section 3.4 for the $P=0.08$ and $P=0.25 \mathrm{CME}$ probabilities, respectively). Note, however, that the $\mathrm{O}_{3}$ loss would likely continue with longer-duration simulated flaring and proton fluence. Based on the simulations in Segura et al. (2010), one does not expect much UVC flux to penetrate to the ground given those column $\mathrm{O}_{3}$ abundances even at higher energies, as one can see in the surface UV for various conditions listed in Table 3. During quiescence, the value of UVB and UVC increases substantially for all simulated cases. At the end of the two 10-year runs in Fig. 11, the UVC reaching the surface at the peak of representative flare events is $\sim 17-$ $298 \mu \mathrm{W} \mathrm{m}{ }^{-2}$. While this level is still $\sim 8-9$ orders of mag- nitude greater than the steady-state, quiescent UVC flux, it is still less than the UVC flux calculated for Earth, 3.9 and $2 \mathrm{Ga}$ ago (Table 6 in Rugheimer et al., 2015; Arney et al., 2016). Based on Earth history, this amount of UVC radiation may not be a substantial detriment to habitability. For a Carringtonsized flare during the period when the $\mathrm{O}_{3}$ column is depressed to $8 \%$ of steady-state value, $\sim 17 \mu \mathrm{W} \mathrm{m}{ }^{-2}$ UVC reaches the surface. For a near AD Leo-sized flare, $10^{33.6} \mathrm{erg}, \sim 298 \mu \mathrm{W}$ $\mathrm{m}^{-2}$ reaches the surface at the peak of the flare. An AD Leo-

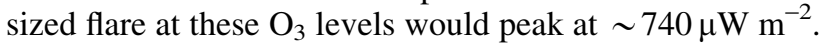

However, as discussed in Section 2.3, the decay phase of the flare dominates the energy input. If one integrates over the temporal evolution applied in the present work, the total amount of UVC energy delivered to the surface for the $10^{33.6}$ erg event (with $8 \%$ equilibrium $\mathrm{O}_{3}$ column) is $0.431 \mathrm{~J}$ $\mathrm{m}^{-2}$ over the $\sim 10^{4} \mathrm{~s}$ event, giving an average UVC flux of $\sim 43.1 \mu \mathrm{W} \mathrm{m}{ }^{-2}$. For the AD Leo event, this figure would be a factor of $\sim 2.5$ larger, due to the increased event amplitude and duration. Obtaining a $90 \%$ kill rate for a commonly studied, radiation-resistant bacterial species, Deinococcus radiodurans, requires a dose of $\sim 550 \mathrm{~J} \mathrm{~m}^{-2}$ (Gascón et al., 1995), which requires more than 1200 of such $10^{33.6}$ erg flare events at an $8 \%$ equilibrium $\mathrm{O}_{3}$ level.

The average energy of all flare events for the 10-year distributions is $\sim 10^{31.4} \mathrm{erg}$, each of which-when integratedprovides a UVC dose of only $\sim 0.13 \mathrm{~mJ} \mathrm{~m}^{-2}$ to the planetary surface with $8 \%$ of Earth's equilibrium $\mathrm{O}_{3}$ column. One should consider the effect to any underlying precursor organic molecules for which UV light may be a driver of complexity (e.g., Beckstead et al., 2016; Rapf and Vaida, 2016; Ranjan et al., 2017), however, regarding habitability. Another consideration is that the $\mathrm{O}_{3}$ column in these cases continues to be eroded.

The bottom panel of Fig. 12 represents a worst-case scenario, where the $\mathrm{O}_{3}$ column has been depleted to $\sim 10^{-4}$ of steady-state equilibrium value. This occurs in our simulations with repeated, extreme flaring with high proton fluence. In the present work, such a state is reached after only 4.9 years, given one AD Leo-sized proton event per month-as seen in the bottom panel of Fig. 8. While such a flare frequency for that energy is not observed from GJ1243 at its current age, early stellar hosts and perhaps more highly active $\mathrm{M}$ dwarf hosts could exhibit such activity for millions of years. It is instructive to quantitatively consider these UV fluxes, as determining these values could offer insight to constraining the early development of complex organics or life on planetary surfaces-not the depths of any ocean, however.

Figure 12, bottom panel, shows the UV flux in $\mathrm{W} \mathrm{m}^{-2} \AA^{-1}$ for four different cases. The cases include quiescent UV flux for the equilibrium $\mathrm{O}_{3}$ column at the TOA and at the surface. In addition, three cases are shown for atmospheric conditions where the $\mathrm{O}_{3}$ column is depleted to a factor of $1.6 \times 10^{-4}$ of equilibrium value-one quiescent case and two flare cases. For purposes of discussion, the flare cases include the UV flux for the most frequent flare energy in the present work, $10^{30.5} \mathrm{erg}$, and the most extreme case for the peak flux of a $10^{34}$ erg flare.

The primary effect of the highly depleted $\mathrm{O}_{3}$ layer is to allow more overall UV flux at the surface, but importantly the increased surface flux is in the UVB and UVC regions $\geq 2000 \AA$ A Even at lower $\mathrm{O}_{3}$ levels, any extreme lower- 


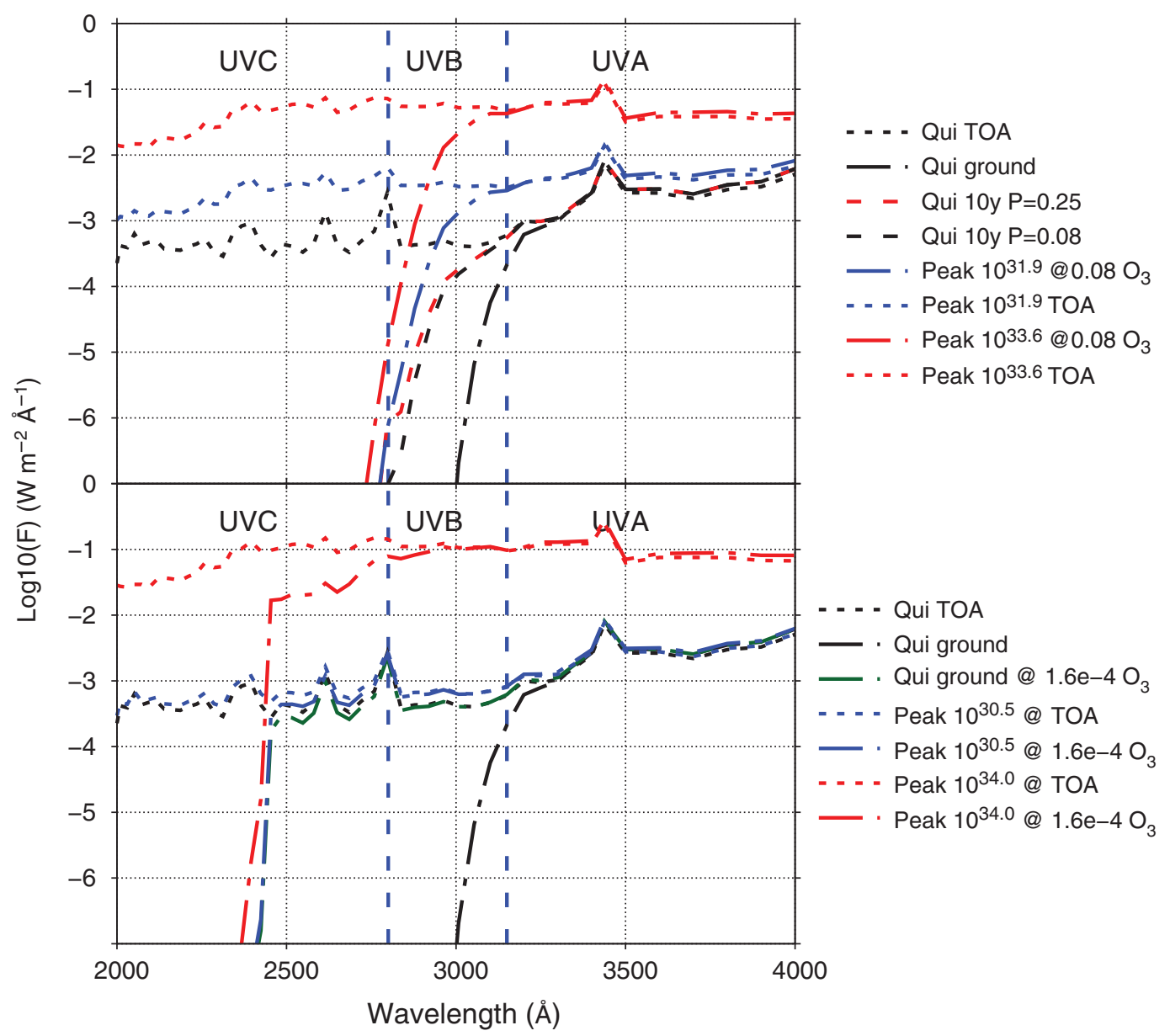

FIG. 12. UV flux for (top) GJ1243 FFD-generated flares and (bottom) extreme $\mathrm{O}_{3}$ loss. Steady-state $\mathrm{O}_{3}$ column at the TOA (dotted black line) and planetary surface (dash-dotted black line); conditions with depleted $\mathrm{O}_{3}$ column at surface (green dash-dotted line); at top (bottom) conditions at the peak of a $10^{31.9}\left(10^{30.5}\right)$ erg flare at TOA (blue dotted line) and surface (blue dash-dotted line); conditions at the peak of a $10^{34}$ erg flare at TOA (red dotted line) and surface (red dashdotted line). Integrated UVC flux values are given in Table 3.

Table 3. Integrated UVC Flux (SEe Fig. 12)

\begin{tabular}{|c|c|c|c|}
\hline & $\begin{array}{c}U V C \text { at } \\
\text { TOA } \\
W m^{-2}\end{array}$ & $\begin{array}{l}U V C \text { at } \\
\text { surface } \\
W m^{-2}\end{array}$ & $\begin{array}{c}\text { Integrated } \\
U V C \\
J^{-2}\end{array}$ \\
\hline $\begin{array}{l}\text { Earth } \\
\text { GJ1243 planet }\end{array}$ & 6.73 & $2.13 \times 10^{-14}$ & - \\
\hline Quiescence, full $\mathrm{O}_{3}$ & 2.76 & $4.15 \times 10^{-13}$ & - \\
\hline Quiescence, $0.1 \mathrm{O}_{3}$ & 2.76 & $1.05 \times 10^{-6}$ & - \\
\hline Quiescence, $0.08 \mathrm{O}_{3}$ & 2.76 & $1.45 \times 10^{-5}$ & - \\
\hline Quiescence, $1.6 \times 10^{-4} \mathrm{O}_{3}$ & 2.76 & 0.18 & - \\
\hline $10^{31.9}$ peak, $0.08 \mathrm{O}_{3}$ & 11.4 & $1.7 \times 10^{-5}$ & $4.5 \times 10^{-3}$ \\
\hline $10^{33.6}$ peak, $0.08 \mathrm{O} 3$ & 180.4 & $2.98 \times 10^{-4}$ & $4.31 \times 10^{-1}$ \\
\hline $10^{30.5}$ peak, $1.6 \times 10^{-4} \mathrm{O}_{3}$ & 3.63 & 0.24 & $3.53 \times 10^{1}$ \\
\hline $10^{34}$ peak, $1.6 \times 10^{-4} \mathrm{O}_{3}$ & 368.76 & 60.8 & $1.27 \times 10^{5}$ \\
\hline
\end{tabular}

UVC flux $(<2800 \AA)$ in $\mathrm{W} \mathrm{m}^{-2}$, integrated over the examples in Fig. 12, at the TOA and the planetary surface for quiescent conditions with steady state $\mathrm{O}_{3}$ column, quiescent conditions with depleted $\mathrm{O}_{3}$ column $\left(0.1,0.08\right.$, and $1.6 \times 10^{-4}$ of equilibrium value), and peak values for $10^{30.5}, 10^{31.9}$, and $10^{34}$ erg flares with associated depleted $\mathrm{O}_{3}$ values. wavelength flux is likely to be absorbed by the $\mathrm{O}_{2}, \mathrm{H}_{2} \mathrm{O}$, and $\mathrm{CO}_{2}$ present in the atmosphere (e.g., Ranjan et al., 2017). We have integrated UVC flux for each of the examples in Fig. 12, the results of which are displayed in Table 3. Note that when the $\mathrm{O}_{3}$ is depleted by a factor of order $10^{4}$, even the quiescent, background spectrum of the $M$ dwarf is sufficient to deliver $0.18 \mathrm{~W} \mathrm{~m}^{-2}$ to the surface of the planet. Given the UV dose required for a germicidal dose, $\sim 10 \mathrm{~J}$ $\mathrm{m}^{-2}$, this indicates that the surface would be sterilized within a few minutes, but likely before the $\mathrm{O}_{3}$ column was depleted to this level. This level of flux does not take into account the likely continuation of frequent flare activity during the high $\mathrm{O}_{3}$ depletion.

During the flare of lowest energy in the present work$10^{30.5}$ - peak UVC flux at the surface is $\sim 30 \%$ higher than during stellar quiescence, at $\sim 0.24 \mathrm{~W} \mathrm{~m}^{-2}$. It is important to note here that approximately seven of these flares occur daily on an active M dwarf like GJ1243 and have duration on the order of tens of minutes. However, even without the presence of these numerous, frequent, low-energy flares, the quiescent value alone is relatively high. During the peak of a large, $\mathrm{AD}$ Leo-like flare, $60.8 \mathrm{~W} \mathrm{~m}^{-2}$ bathes the planetary 
surface; over the course of the multi-hour flare, that value ranges from $\sim 1 \%$ of that flux up to the peak value. In particular, the rise and decay phases of our simulated flare last $\sim 4 \mathrm{~h}$, delivering $\sim 127 \mathrm{~kJ} \mathrm{~m}^{-2}$ of UVC to the surface during that time, compared to $35.3 \mathrm{~J} \mathrm{~m}^{-2}$ for the smaller $10^{30.5}$ erg flares that occur several times daily. In these cases, the survival of even the hardiest of known bacterial species is in question.

As pointed out by Ranjan et al. (2017), laboratory experiments are needed to evaluate how UV fluxes like the ones expected at planets in the habitable zone of $\mathrm{M}$ dwarfs may influence the construction of complex organic molecules. However, if such UV fluxes are detrimental to building complex organic molecules, below approximately $9 \mathrm{~m}$ of seawater, this photodissociating and photoionizing radiation will not penetrate, allowing such complexity to flourish where there is a sufficient free energy gradient and raw materials, for example, around hydrothermal vents (e.g., Kiang et al., 2007).

\subsection{The effect of flare-driven atmospheric evolution on observation}

Because stellar activity can modify a terrestrial planet's atmospheric composition, it can also affect observable characteristics of the planet. Upcoming missions such as the James Webb Space Telescope (JWST), and mission concepts such as the Large UV Optical Infrared Survey Telescope (LUVOIR), will have the capabilities to probe terrestrial exoplanet atmospheres using transmission and direct imaging spectroscopy, respectively. These observations will enable a direct comparison of the impact of $\mathrm{M}$ dwarf stellar activity on terrestrial atmospheric evolution, when compared to the evolution and atmospheric characteristics of Earth. Models like those described here, paired with knowledge of the activity levels and behavior of the parent star, can be used to accurately interpret observed spectra with respect to habitability and can also be used to determine how atmospheres might be altered by the presence of life. Similarly, modeling can provide the stellar and atmospheric context necessary to correctly identify spectral features due to abiotic false positives for $\mathrm{O}_{2}$ (Harman et al., 2015; Schwieterman et al., 2016; Meadows, 2017).

One particular strong atmospheric signal pointing to biotic chemical disequilibrium on Earth is the coexistence of abundant $\mathrm{N}_{2}, \mathrm{O}_{2}$, and $\mathrm{H}_{2} \mathrm{O}$ (Krissansen-Totton et al., 2016). In the present work, the levels of these particular species are not affected in either the 100 template flare simulations (from Section 3.4, see Fig. 13) or the 10-year real-CME simulations (from Section 3.4, see Fig. 14). However, it is noted that for our simulated starting point of an Earth-like planet, other species of interest that might be targeted by transmission spectroscopy can be altered significantly. For instance, the $\mathrm{CO}_{2}$ in the upper atmosphere is notably increased by the photolysis of $\mathrm{CH}_{4}$ driven by flaring activity, by $\geq 50 \mathrm{ppm}$ at altitudes of $45 \mathrm{~km}$ and above, as seen in Figs. 13 and 14. Similarly, the $\mathrm{CH}_{4}$ levels have been depleted by similar amounts. $\mathrm{O}_{3}$ obviously experiences significant reduction, and $\mathrm{H}_{2} \mathrm{O}$ is altered.

As the more realistic simulations in Fig. 14 are artificially cut off after a maximum of 10 years, we do not show the result of the anticipated millions of years of stellar activity on the atmospheric state. In the lower panels of Fig. 14, one can see that the $\mathrm{O}_{2}$ levels have been slightly eroded, by $\sim 0.1 \%$. While this is unlikely to produce significant alterations of spectral transit depth, continued reduction of $\mathrm{O}_{2}$ over the periods of stellar activity exhibited by $\mathbf{M}$ dwarf hosts could potentially drive the signal below threshold for detection, giving a false indicator of the atmospheric state near the surface.

Our simulations indicate that a planet with an Earth-like atmosphere subjected to GJ1243 levels of flare and proton event activity is unlikely to be altered to the point of obscuring the strong $\mathrm{N}_{2}-\mathrm{O}_{2}-\mathrm{H}_{2} \mathrm{O}$ chemical disequilibrium present. However, even on the short term of the present 10-year simulations, $\mathrm{O}_{3}$ is rapidly eroded by multiple orders of magnitude, which indicates the possibility that no ozone layer may exist on such cases even if there is life producing oxygen, and there exists indication in the present simulations that $\mathrm{O}_{2}$ could be further reduced by extended periods of $\mathrm{M}$ dwarf activity (see, e.g., Fig. 13). Further work is required to explore the details of this particular consequence.

\subsection{Other M dwarf hosts}

We have focused here on $\mathrm{M}$ dwarf activity observed on GJ1243. M dwarf stellar activity is highly variant, however, with GJ1243 only a moderately active star. Other active hosts, such as GJ876, display a drastically lower level of activity and anticipated total proton flux (e.g., Youngblood et al., 2017). Even with the lower levels of event-specific proton flux estimated for GJ876 $\left(10^{2}\right.$ to $10^{3}$ pfu compared to the events from $10^{4}$ to $10^{8}$ in the present work), one finds that the long-term stability of $\mathrm{O}_{3}$ is reduced on a relatively quick timescale, to $\sim 80-90 \%$ of the equilibrium value after 40 months of flaring (see, e.g., Fig. 11 in Youngblood et al., 2017). Extrapolating that rate of loss leads to $\geq 99 \% \mathrm{O}_{3}$ depletion after $10^{9}$ to $10^{13} \mathrm{~s}$ ( $\sim 10^{2}$ to $10^{5}$ years $)$, though as seen in Fig. 8, it is possible the atmosphere reaches a new equilibrium $\mathrm{O}_{3}$ column.

Similarly, there are $\mathrm{M}$ dwarfs capable of much higher levels of activity, with very active M3-M5 stars observed displaying levels of activity from 1-2 orders of magnitude higher than what we have used in the present work (e.g., Hilton, 2011). Given the level of $\mathrm{O}_{3}$ depletion seen in the present work, it is likely that planets orbiting more active hosts show a more rapid, more thorough destruction of any potential $\mathrm{O}_{3}$ layer.

\subsection{Recently observed Earth-like planets}

Recent observations have discovered potentially habitable worlds orbiting both Proxima Centauri and TRAPPIST-1, M6 and M8 stellar hosts, respectively (Anglada-Escudé et al., 2016; Gillon et al., 2017). MOST observations have indicated that Proxima Centauri is highly active, with multiple flares per day at $10^{29.5} \mathrm{erg}$, up to a $10^{31.5} \mathrm{erg}$ event every week (Davenport et al., 2016). This level of activity could drive significant loss of $\mathrm{O}_{3}$ (see, e.g., middle panel of Fig. 8), especially given that the planet orbits at $\sim 0.0485$ AU. The closer orbit increases the expected proton fluence and UV flux by an order of magnitude, compared to that in the present work-which assumes the planet orbits at 0.16 AU. Balancing this is the lower-energy flare distribution, 


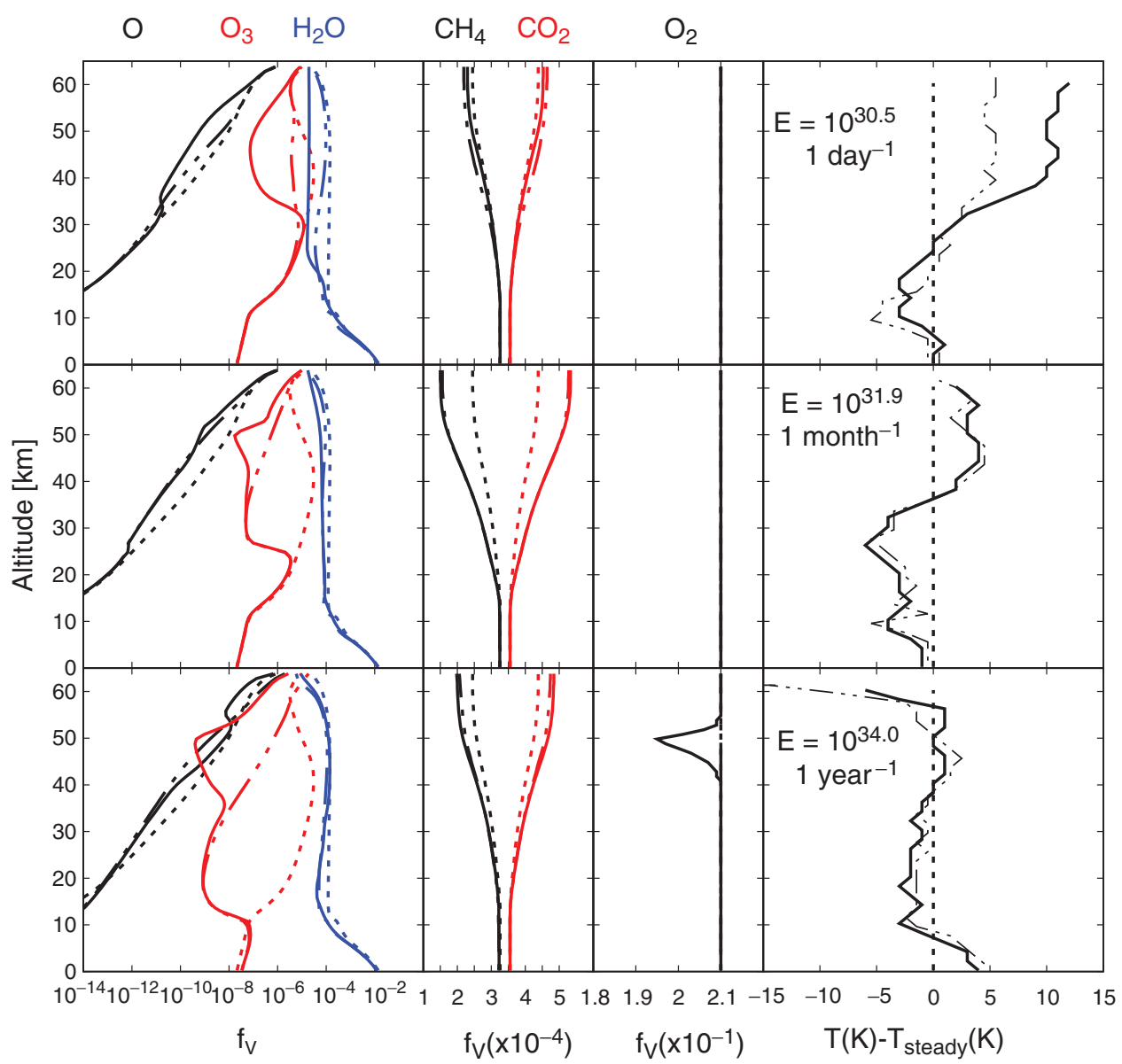

FIG. 13. Atmospheric mixing ratio $\left(f_{V}\right)$ and temperature profiles for the three parameter studies of 100 flares of $\log (E)$ 30.5 at frequency of 1 per day (top row), 31.9 with frequency of 1 per month (middle row), and 34.0 with frequency 1 per year (bottom row). In all cases, the dashed line represents initial steady state, the dash-dotted line signifies the state at the end of the flaring (before recovery), and the solid line represents the state at peak ozone loss during recovery.

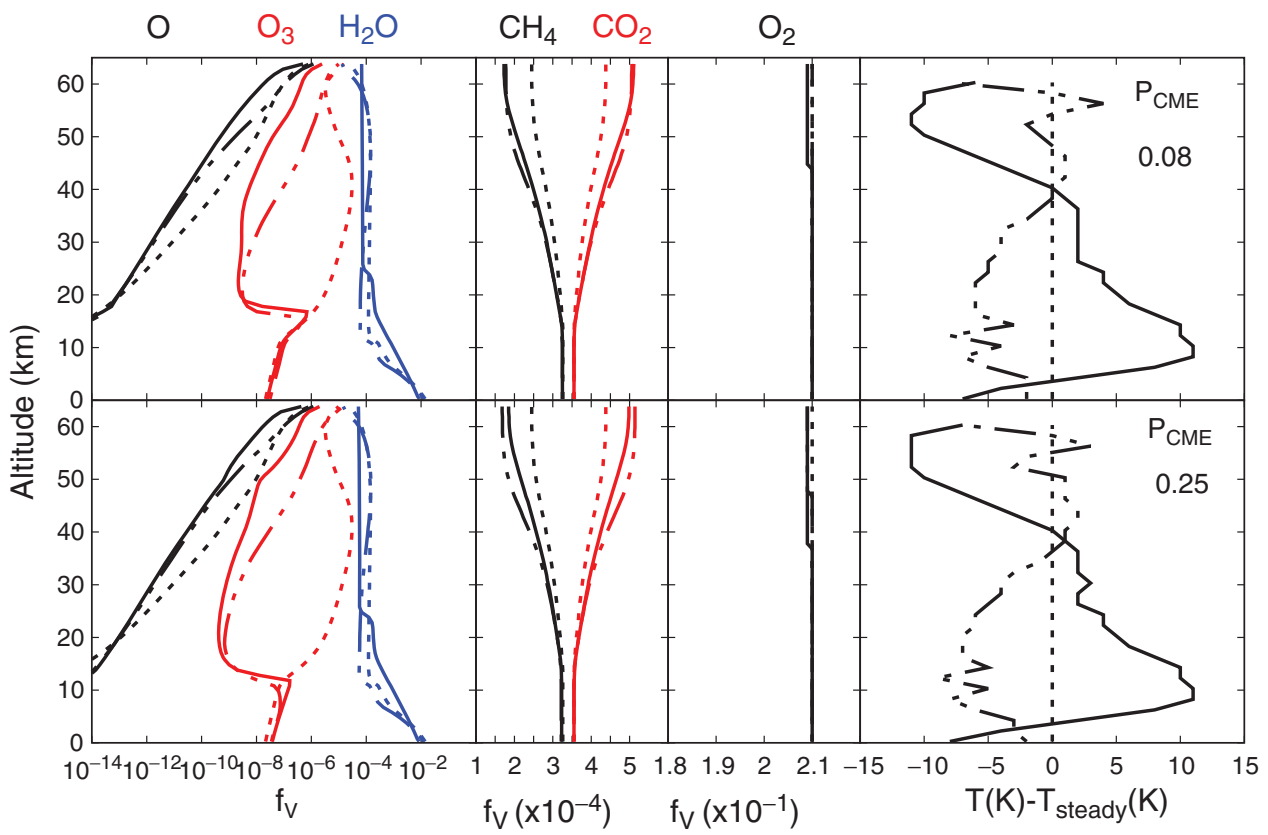

FIG. 14. Atmospheric mixing ratio $\left(f_{V}\right)$ and temperature for two 10-year periods of flare activity, generated from the GJ1243 FFD. The top row is with a conservative CME impact probability of 0.08 , and the bottom row is the more permissive probability of 0.25 . In all cases, the dashed line represents initial steady state, the dash-dotted line signifies the state at the end of the flaring (before recovery), and the solid line represents the state at peak ozone loss during recovery. 
which is lower by about an order of magnitude, which by the scaling used in the present work roughly produces similar fluences at the planet as the orbital and energy scaling cancel each other-though the UV flux would still increase from the isotropic scaling. Therefore, the estimates in Fig. 11 can loosely be ascribed to an Earth-like atmosphere on Proxima Centauri b, but detailed modeling is required to obtain accurate results.

TRAPPIST-1 seems to currently exhibit infrequent flaring activity by optical flux measurements. Vida et al. (2017) used K2 light curves to measure the flare activity of the star and estimated events of order $10^{30}$ to $10^{33} \mathrm{erg}$ occur from once every few days for the lower-energy events to approximately once per 100 days on the high end of the energy range. O'Malley-James and Kaltenegger (2017) showed that the surface habitability of the planets in TRAPPIST-1's habitable zone are constrained by the activity level of the star and oxic state of the atmosphere. There are currently no constraints on the biologically relevant UV flux for TRAPPIST-1, but from Lyman $\alpha$ chromospheric observations and the optical observations above, it is likely the star is active in that wavelength range.

If the surface habitability of these bodies, and those similar that are discovered in the future, is to be assessed, the effect of $\mathrm{M}$ dwarf flaring on any present atmosphere must be explored. In the present work, we assumed the starting point of an Earth-like atmosphere, which presupposes a high $\mathrm{O}_{2}$ content and chemical disequilibrium driven by life. However, it is likely that observed planets consist of varied compositions that will require detailed modeling based on observation from future missions such as JWST or LUVOIR.

\subsection{Anoxic, hazy atmospheres}

It is important to recognize that hazy layers or the relatively high population of aerosols could change these findings, as they are efficient absorbers of UV radiation. In an early-Earth-type anoxic atmosphere, Arney et al. (2016) showed that the presence of haze can cut the incident UVC radiation at the surface of the planet by a factor of $\sim 30$ with a solar zenith angle of $60^{\circ}$. While we cannot observe the conditions of early Earth, we do have a decent laboratory in the Solar System for the formation and evolution of atmospheric hazes: Saturn's satellite Titan. It has been observed that energetic ions from Saturn's magnetosphere can drive significant changes in ionospheric upper atmospheric chemistry (e.g., Cravens et al., 2008); such activity is thought to significantly contribute to the formation of high-atomic-massunit organic haze layers that can then precipitate and coalesce into tholins at the surface of the satellite (e.g., Waite et al., 2007; Lavvas et al., 2013).

Simulating the effects of stellar activity and proton chemistry on a terrestrial atmosphere after the development of photosynthesis but before the "great oxidation event" could provide further insight into the shielding efficiency of aerosols and hazes during this early stage of the development of life, and therefore identify the amount of stress placed on an unshielded, early biosphere. Given increased activity during early solar evolution, and from $\mathrm{M}$ dwarfs in general, it is important to investigate how the presence of energetic proton precipitation would affect the formation and sustained chemistry of high-altitude haze layers, and therefore their impact on UVC radiation at the surface of a potentially habitable planet.

\subsection{Unmagnetized planet}

There is uncertainty in the ability of a global magnetic field to protect against the erosion of terrestrial planetary atmospheres by a stellar wind. On one hand, the magnetized Earth has retained a relatively thick atmosphere while (mostly) unmagnetized Mars has not; on the other hand, unmagnetized Venus has a $\sim 93$ bar atmosphere and experiences approximately twice the solar wind energy deposition per unit area as Earth due to its closer orbital distance.

Similarly, there is a question related to the present work on the ability of a planetary magnetic field to protect the atmosphere from energetic proton deposition by the stellar wind. One might naïvely assume that the lack of a global field allows the protons from the stellar wind free reign to penetrate deeply into the upper atmosphere of such a planet. However, photoionization of the upper atmosphere leads to a spherical, conducting shell (ionosphere) that deflects the magnetized stellar wind. It has been suggested that for Venus that solar wind penetration is not expected to drive significant chemical or physical changes to the ionosphere or lower layers of the atmosphere (e.g., Gombosi et al., 1980). Interestingly, for planets without a global magnetic field that orbit close to their host stars, the increased XUV/EUV flux in the upper atmosphere is expected to produce an ionosphere of higher density and scale height, leading to deflection of the stellar wind at higher altitudes (e.g., Cohen et al., 2015). However, this expansion could lead to more rapid atmospheric erosion, which over time could reduce the strength of the ionospheric deflection. The penetration of energetic protons, like those from CME/SEP events, is still an open question for potentially habitable exoplanetary atmospheres.

For an undisturbed Earth-like geomagnetic field, protons require an energy of $\geq 10 \mathrm{GeV}$ to penetrate directly into the subtropical to equatorial ionosphere (e.g., Rodger et al., 2006). While protons with these energies have been observed from high-energy solar events, the indicate fluxes of $\geq 10 \mathrm{GeV}$ protons are of factor $\geq 6$ orders of magnitude lower than fluxes of protons of $\sim 100 \mathrm{MeV}$ (e.g., Cliver and Dietrich, 2013). However, even if these particles do not penetrate completely to the upper atmosphere, they can become temporarily trapped in the geomagnetic field, and energized protons flowing within the magnetosphere are capable of precipitation into the upper atmosphere, for example, via pitch angle scattering.

The penetration energy requirement also declines with increasing magnetic latitude for a dipole magnetic field (e.g., Shea and Smart, 1992; Smart et al., 2000). There are open regions at the separation between open and closed magnetospheric field lines, that is, the cusp regions, where energetic protons could flow directly from the stellar wind into the upper atmosphere. Further, it is worth noting the geometry, orientation, and magnitude of a planetary field; an inclined dipole field could allow direct proton access to the upper atmosphere, and a strong quadrupole moment, for instance, has polar regions similar to the dipole field geometry of Earth, but also a circum-equatorial region with intersecting field lines where precipitation could occur. 
The interactions between stellar wind, and energetic stellar outflows, are complex combinations of magnetic star-planet interactions that require further study. This begs the question of how effective magnetic fields are at deflecting (non-)relativistic proton events, shielding the planetary atmosphere. We plan to investigate and address these issues in Part 2, a companion paper to the present work.

\section{Conclusions}

The conditions under which life can develop are not certain, but clement conditions must be sufficiently stable for sustained development of organic complexity. For $\mathrm{M}$ dwarf-orbiting planets, it is clear they will experience a high flux of electromagnetic and particle radiationunderstanding these effects on the atmosphere is imperative to predicting the likelihood of surface life. As shown in the present work, even with conservative assumptions, $M$ dwarf activity can drive extreme loss in the $\mathrm{O}_{3}$ column for an Earth-like planet within a period of time that is several orders of magnitude shorter than the known active lifetimes for low-mass stars. Absent other shielding, short wavelength UVB and UVC will blanket the surface. The effects of such radiation can be harmful to complex organic structures, but there is much study to be done to reproduce such an environment to understand how life could emerge.

In particular, we find:

- EM-only flaring for a stellar host like GJ1243 is unlikely to drive significant $\mathrm{O}_{3}$ loss, with only $\sim 37 \%$ $\mathrm{O}_{3}$ column loss by the present age of the Universe for the modeled flaring activity. In contrast, the same loss can be achieved in a minimum $\sim 1.3$ years by weekly proton impacts from the lowest-energy events simulated.

- $\mathrm{O}_{3}$ column loss by flaring parameters from an observed host like GJ1243, as shown in Fig. 11, can drive $\geq 99.9 \%$ destruction of the $\mathrm{O}_{3}$ column in as little as $0.5 \mathrm{Myr}$, with a trend of increasing loss that is unlikely to reverse.

- The impact of one AD Leo-sized flare per month is sufficient to drive a loss of $99.99 \%$ of $\mathrm{O}_{3}$ column within $\sim 8$ years. In this case, the surface of a planet will experience quiescent UVC flux of $\sim 0.18 \mathrm{~W} \mathrm{~m}^{-2}$, with subsequent $10^{34}$ flares delivering $\sim 127 \mathrm{~kJ} \mathrm{~m}^{-2}$ of UVC for a single event. Further experiments are needed to evaluate the impact of these fluxes on the onset of complex organic chemistry and life.

- In addition to reducing the $\mathrm{O}_{3}$ mixing ratio by orders of magnitude, our results suggest that atmospheric species of interest (e.g., $\mathrm{CO}_{2}, \mathrm{CH}_{4}$ ) can be modified to levels that will be observable by upcoming missions. Mixing ratio vertical profiles change slightly for steady state conditions, while the high level of stellar activity can drive long-term profile changes of at least $\sim 100 \mathrm{ppm}$ within the span of decades.

Future efforts should include detailed simulations of varying magnetospheric conditions on deflection of proton events to gain an understanding of how well $\mathrm{M}$ dwarforbiting planets are protected by an intrinsic magnetic dipole. Of further interest is developing simulations of atmospheric effects of $\mathrm{M}$ dwarf activity for planets with non-Earth-like starting conditions, for example, high $\mathrm{CO}_{2}$, high $\mathrm{O}_{2}$.

\section{Acknowledgments}

The authors thank two anonymous reviewers for their comments and suggestions that greatly improved this manuscript. M.T. would like to thank Erika Harnett, Joshua Krissansen-Totten, and Eddie Schwieterman for helpful discussions, and acknowledges the generous support of the NAI Virtual Planetary Laboratory and University of Washington Astrobiology Program. This material is based upon work performed by the NASA Astrobiology Institute's Virtual Planetary Laboratory Lead Team, supported by the National Aeronautics and Space Administration through the NASA Astrobiology Institute under Cooperative Agreement number NNA13AA93A. We acknowledge use of NASA/ GSFC's Space Physics Data Facility's OMNIWeb (or CDAWeb or ftp) service, and OMNI data. We also acknowledge the use of GOES data at NOAA's National Centers for Environmental Information (NCEI) Space Weather data access.

A.S. also acknowledges support from UNAM PAPIIT project IN109015.

\section{References}

Airapetian, V.S., Glocer, A., Khazanov, G.V., Loyd, R.O.P., France, K., Sojka, J., Danchi, W.C., and Liemohn, M.W. (2017) How hospitable are space weather affected habitable zones? The role of ion escape. Astrophys $J$ 836:L3.

Anglada-Escudé, G., Amado, P.J., Barnes, J., Berdiñas, Z.M., Butler, R.P., Coleman, G.A., de la Cueva, I., Dreizler, S., Endl, M., Giesers, B. Jeffers, S.V., Jenkins, J.S., Jones, H.R., Kiraga, M., Kürster, M., López-González, M.J., Marvin, C.J., Morales., N., Morin, J., Nelson, R.P., Oritz, J.L., Ofir, A., Paardekooper, S.-J., Reiners, A., Rodríguez, E., RodríguezLópez, C., Sarmiento, L.F., Strachan, J.P., Tsapras, Y., Tuomi, M., and Zechmeister, M. (2016) A terrestrial planet candidate in a temperate orbit around Proxima Centauri. Nature 536:437-440.

Arney, G., Domagal-Goldman, S.D., Meadows, V.S., Wolf, E.T., Schwieterman, E., Charnay, B., Claire, M., Hébrard, E., and Trainer, M.G., (2016) The pale orange dot: the spectrum and habitability of hazy Archean Earth. Astrobiology 16:873-899.

Beckstead, A.A., Zhang, Y., de Vries, M.S., and Kohler, B. (2016) Life in the light: nucleic acid photoproperties as a legacy of chemical evolution. Phys Chem Chem Phys 18: 24228-24238.

Belov, A., Garcia, H., Kurt, V., Mavromichalaki, H., and Gerontidou, M. (2005) Proton enhancements and their relation to the X-ray flares during the three last solar cycles. Solar Physics 229:135-159.

Cliver, E.W. and Dietrich, W.F. (2013) The 1859 space weather event revisited: limits of extreme activity. Journal of Space Weather and Space Climate 3, doi:10.1051/swsc/2013053.

Cohen, O., Ma, Y., Drake, J.J., Glocer, A., Garraffo, C., Bell, J.M., Gombosi, T.I. (2015) The interaction of Venus-like, Mdwarf planets with the stellar wind of their host star. Astrophys $J$ 806, doi:10.1088/0004-637X/806/1/41.

Cravens, T.E., Robertson, I.P., Ledvina, S.A., Mitchell, D., Krimigis, S.M., and Waite, J.H. (2008) Energetic ion precipitation at Titan. Geophys Res Lett 35, doi:10.1029/ 2007 GL032451.

Crosby, N.B., Aschwanden, M.J., and Dennis, B.R. (1993) Frequency distributions and correlations of solar X-ray flare parameters. Solar Physics 143:275-299. 
Davenport, J.R., Hawley, S.L., Hebb, L., Wiseniewski, J.P., Kowalski, A., Johnson, E.C., Malatesta, M., Peraza, J., Keil, M., Silverberg, S.M., Jansen, T.C., Scheffler, M.S., Berdis, J.R., Larsen, D.M., and Hilton, E.J. (2014) Kepler flares. II. The temporal morphology of white-light flares on GJ 1243. Astrophys J 797, doi:10.1088/0004-637X/797/2/122.

Davenport, J.R., Kipping, D.M., Sasselov, D., Matthews, J.M., and Cameron, C. (2016) MOST observations of our nearest neighbor: flares on Proxima Centauri. Astrophys $J$ 829, doi: 10.3847/2041-8205/829/2/L31.

De Mora, S., Demers, S., and Vernet, M. (2000) The Effects of $U V$ Radiation in the Marine Environment, Cambridge Environmental Chemistry Series Vol. 10, Cambridge University Press, Cambridge, UK.

Dierckxsens, M., Tziotziou, K., Dalla, S., Patsou, I., Marsh, M. S., Crosby, N. B., Malandraki, O., Tsiropoula, G. (2015) Relationship between solar energetic particles and properties of flares and CMEs: statistical analysis of solar cycle 23 events. Solar Physics 290:841-874.

Dole, S.H. (1964) Habitable Planets for Man, Blaisdell, New York.

Dong, C., Jin, M., Lingam, M., Airapetian, V.S., Ma, Y., and van der Holst, B. (2018) Atmospheric escape from the TRAPPIST-1 planets and implications for habitability. Proc Natl Acad Sci USA 115:260-265.

Ejzak, L.M., Melott, A.L., Medvedev, M.V., and Thomas, B.C. (2007) Terrestrial consequences of spectral and temporal variability in ionizing photon events. Astrophys $J$ 654: 373-384.

Estrela, R. and Valio, A. (2017) Superflare UV flashes impact on Kepler-96 system: a glimpse of habitability when the ozone layer first formed on Earth. arXiv:1708.05400

France, K., Linsky, J.L., Tian, F., Froning, C.S., and Roberge, A. (2012) Time-resolved ultraviolet spectroscopy of the M dwarf GJ876 exoplanetary system. Astrophys J 750:L32.

France, K., Froning, C.S., Linsky, J.L., Roberge, A., Stocke, J.T., Tian, F., Bushinsky, R., Désert, J.-M., Mauas, P., Vieytes, M., and Walkowicz, L. (2013) The ultraviolet radiation environment around $\mathrm{M}$ dwarf exoplanet host stars. Astrophys $J$ 763, doi:10.1088/0004-637X/763/2/149.

Garcia-Sage, K., Glocer, A., Drake, J.J., Gronoff, G., and Cohen, O. (2017) On the magnetic protection of the atmosphere of Proxima Centauri b. Astrophys J 844, doi:10.3847/20418213/aa7eca.

Garraffo, C., Drake, J.J., and Cohen, O. (2016) The space weather of Proxima Centauri b. Astrophys J 833:L4.

Garraffo, C., Drake, J.D., Cohen, O., Alvarado-Gomez, J.D., and Moschou, S.P. (2017) The threatening magnetic and plasma environment of the TRAPPIST-1 planets. Astrophys $J$ 843, doi:10.3847/2041-8213/aa79ed.

Gascón, J., Oubiña, A., Pérez-Lezaun, A., and Urmeneta, J. (1995) Sensitivity of selected bacterial species to UV radiation. Curr Microbiol 30:177-182.

Gillon, M., Jehin, E., Lederer, S.M., Delrez, L., de Wit, J., Burdanov, A., Van, Grootel, V., Burgasser, A.J., Triaud, A.H., Opitom, C., Demory, B.O., Sahu, D.K., Bardalez Gagliuffi, D., Magain, P, and Queloz, D. (2016) Temperate Earth-sized planets transiting a nearby ultracool dwarf star. Nature 533:221-224.

Gillon, M., Triaud, A.H., Demory, B.O., Jehin, E., Agol, E., Deck, K.M., Lederer, S.M., de Wit, J., Burdanov, A., Ingalls, J.G., Bolmont, E., Leconte, J., Raymond, S.N., Selsis, F., Turbet, M., Barkaoui, K., Burgasser, A., Burleigh, M.R., Carey, S.J., Chaushev, A., Copperwheat, C.M., Delrez, L.,
Fernandes, C.S., Holdsworth, D.L., Kotze, E.J., Van Grootel, V., Almleaky, Y., Benkhaldoun, Z., Magain, P., and Queloz, D. (2017) Seven temperate terrestrial planets around the nearby ultracool dwarf star TRAPPIST-1. Nature 542:456-460.

Giorgi, F. and Chameides, W. (1985) The rainout parameterization in a photochemical model. J Geophys Res Atmospheres 90:7872-7880.

Gombosi, T., Cravens, T., Nagy, A., Elphic, R., and Russell, C. (1980) Solar wind absorption by Venus. J Geophys Res Space Physics 85:7747-7753.

Gopalswamy, N., Yashiro, S., Thakur, N., Mäkelä, P., Xie, H., and Akiyama, S. (2016) The 2012 July 23 backside eruption: an extreme energetic particle event? Astrophys $J$ 833, doi: 10.3847/1538-4357/833/2/216.

Harman, C.E., Schwieterman, E.W., Schottelkotte, J.C., and Kasting, J.F. (2015) Abiotic $\mathrm{O}_{2}$ levels on planets around F, G, $\mathrm{K}$, and M stars: possible false positives for life? Astrophys $J$ 812, doi:10.1088/0004-637X/812/2/137.

Hawley, S.L. and Pettersen, B.R. (1991) The great flare of 1985 April 12 on AD Leonis. Astrophys J 378:725-741.

Hawley, S.L., Fisher, G.H., Simon, T., Cully, S.L., Deustua, S.E., Jablonski, M., Johns-Krull, C.M., Pettersen, B.R., Smith, V., Spiesman, W.J., and Valenti, J. (1995) Simultaneous extremeultraviolet Explorer and optical observations of AD Leonis: evidence for large coronal loops and the Neupert effect in stellar flares. Astrophys J 453, doi:10.1086/176408.

Hawley, S.L., Davenport, J.R., Kowalski, A.F., Wisniewski, J.P., Hebb, L., Deitrick, R., and Hilton, E.J. (2014) Kepler Flares. I. Active and inactive M dwarfs. Astrophys J 797, doi: 10.1088/0004-637X/797/2/121.

Hilton, E. (2011) The Galactic M Dwarf Flare Rate. PhD thesis, University of Washington, Seattle, WA.

Hudson, H.S. (2011) Global properties of solar flares. Space Science Reviews 158:5-41.

Joshi, M. (2003) Climate model studies of synchronously rotating planets. Astrobiology 3:415-427.

Joshi, M.M., Haberle, R.M., and Reynolds, R.T. (1997) Simulations of the atmospheres of synchronously rotating terrestrial planets orbiting M dwarfs: conditions for atmospheric collapse and the implications for habitability. Icarus 129:450-465.

Kasting, J.F. and Ackerman, T.P. (1986) Climatic consequences of very high carbon dioxide levels in the Earth's early atmosphere. Science 234:1383-1385.

Kay, C., Opher, M., and Kornbleuth, M. (2016) Probability of CME impact on exoplanets orbiting $M$ dwarfs and solar-like stars. Astrophys $J$ 826, doi:10.3847/0004-637X/826/2/195.

Khodachenko, M.L., Ribas, I., Lammer, H., Grießmeier, J.-M., Leitner, M., Selsis, F., Eiroa, C., Hanslmeier, A., Biernat, H.K., Farrugia, C.J., and Rucker, H.O. (2007) Coronal mass ejection (CME) activity of low-mass $\mathrm{M}$ stars as an important factor for the habitability of terrestrial exoplanets. I. CME impact on expected magnetospheres of Earth-like exoplanets in close-in habitable zones. Astrobiology 7:167-184.

Kiang, N.Y., Segura, A., Tinetti, G., Govindjee, Blankenship, R.E., Cohen, M., Siefert, J., Crisp, D., and Meadows, V.S. (2007) Spectral signatures of photosynthesis. II. Coevolution with other stars and the atmosphere on extrasolar worlds. Astrobiology 7:252-274.

Kowalski, A.F., Hawley, S.L., Wisniewski, J.P., Osten, R.A., Hilton, E.J., Holtzman, J.A., Schmidt, S.J., and Davenport, J.R.A. (2013) Time-resolved properties and global trends in dMe flares from simultaneous photometry and spectra. Astrophys J Supp Ser 207, doi:10.1088/0067-0049/207/1/15. 
Krissansen-Totton, J., Bergsman, D., and Catling, D.C. (2016) On detecting biospheres from chemical thermodynamic disequilibrium in planetary atmospheres. Astrobiology 16:39-67.

Lario, D. and Decker, R. (2011) Estimation of solar energetic proton mission-integrated fluences and peak intensities for missions traveling close to the Sun. Space Weather 9, doi: 10.1029/2011SW000708.

Lavvas, P., Yelle, R. V., Koskinen, T., Bazin, A., Vuitton, V., Vigren, E., Galand, M., Wellbrock, A., Coates, A.J., Wahlund, J.-E., Crary, F.J., and Snowden, D. (2013) Aerosol growth in Titan's ionosphere. Proc Natl Acad Sci USA 110:2729-2734.

Luger, R. and Barnes, R. (2015) Extreme water loss and abiotic $\mathrm{O}_{2}$ buildup on planets throughout the habitable zones of $\mathrm{M}$ dwarfs. Astrobiology 15:119-143.

Meadows, V.S. (2017) Reflections on $\mathrm{O}_{2}$ as a biosignature in exoplanetary atmospheres. Astrobiology 17:1022-1052.

Meadows, V.S., Arney, G.N., Schwieterman, E.W., LustigYaeger, J., Lincowski, A.P., Robinson, T., DomagalGoldman, S.D., Deitrick, R., Barnes, R.K., Fleming, D.P., Luger, R., Driscoll, P.E., Quinn, T.R., and Crisp, D. (2018a) The habitability of Proxima Centauri b: environmental states and observational discriminants. Astrobiology 18:133-189.

Meadows, V.S., Reinhard, C.T., Arney, G.N., Parenteau, M.N., Schwieterman, E.W., Domagal-Goldman, S., Lincowski, A.P., Stapelfeldt, K.R., Rauer, H., DasSarma, S., Hegde, S., Narita, N., Deitrick, R., Lustig-Yaeger, J., Lyons, T.W., Siegler, N., and Grenfell, J.L. (2018b) Exoplanet biosignatures: understanding oxygen as a biosignature in the context of its environment. Astrobiology 18:630-662.

Mekhaldi, F., Muscheler, R., Adolphi, F., Aldahan, A., Beer, J., McConnell, J.R., Possnert, G., Sigl, M., Svensson, A., Synal, H.A., Welten, K.C., and Woodruff, T.E. (2015) Multiradionuclide evidence for the solar origin of the cosmic-ray events of AD 774/5 and 993/4. Nat Commun 6, doi:10.1038/ ncomms9611.

Melott, A.L., Thomas, B.C., Kachelriess, M., Semikoz, D.V., and Overholt, A.C. (2017) A supernova at 50 pc: effects on the Earth's atmosphere and biota. Astrophys $J$ 840, doi: 10.3847/1538-4357/aa6c57.

Mewaldt, R.A., Cohen, C.M.S., Labrador, A.W., Leske, R.A., Mason, G.M., Sedai, M.I., Looper, M.D., Mazur, J.E., Selesnick, R.S., and Haggerty, D.K. (2005) Proton, helium, and electron spectra during the large solar particle events of October-November 2003. J Geophys Res Space Phys doi:110, 10.1029/2005JA011038.

Mitra-Kraev, U., Harra, L., Güdel, M., Audard, M., BranduardiRaymont, B., Kay, H.R.M., Mewe, R., Raassen, A.J.J., and van Driel-Gesztelyi, L. (2005) Relationship between X-ray and ultraviolet emission of flares from dMe stars observed by XMM-Newton. Astron Astrophys 431:679-686.

O'Malley-James, J.T. and Kaltenegger, L. (2017) UV surface habitability of the TRAPPIST-1 system. Monthly Notices of the Royal Astronomical Society: Letters 469:L26-L30.

Porter, H., Jackman, C., and Green, A. (1976) Efficiencies for production of atomic nitrogen and oxygen by relativistic proton impact in air. J Chem Phys 65, doi:10.1063/1.432812.

Ranjan, S., Wordsworth, R., and Sasselov, D. D. (2017) The surface UV environment on planets orbiting $M$ dwarfs: implications for prebiotic chemistry and the need for experimental follow-up. ApJ 843, doi:10.3847/1538-4357/aa773e.

Rapf, R.J. and Vaida, V. (2016) Sunlight as an energetic driver in the synthesis of molecules necessary for life. Phys Chem Chem Phys 18:20067-20084.
Ribas, I., Bolmont, E., Selsis, F., Reiners, A., Leconte, J., Raymond, S.N., Engle, S.G., Guinan, E.F., Morin, J., Turbet, M., Forget, F., and Anglada-Escudé, G. (2016) The habitability of Proxima Centauri b-I. Irradiation, rotation and volatile inventory from formation to the present. Astron Astrophys 596:A111.

Rodger, C.J., Clilverd, M.A., Verronen, P.T., Ulic, R., Jarvis, M.J., and Turunen, E. (2006) Dynamic geomagnetic rigidity cutoff variations during a solar proton event. J Geophys Res Space Phys 111, doi:10.1029/2005JA011395.

Rodger, C.J., Verronen, P.T., Clilverd, M.A., Seppälä, A., and Turunen, E. (2008) Atmospheric impact of the Carrington event solar protons. J Geophys Res: Atmospheres 113, doi: 10.1029/2008JD010702.

Rugheimer, S., Segura, A., Kaltenegger, L., and Sasselov, D. (2015) UV surface environment of Earth-like planets orbiting FGKM stars through geological evolution. Astrophys $J$ 806, doi:10.1088/0004-637X/806/1/137.

Rusch, D.W., Gerard, J.-C., Solomon, S., Crutzen, P.J., and Reid, G.C. (1981) The effect of particle precipitation events on the neutral and ion chemistry of the middle atmosphereI. Odd nitrogen. Planet Space Sci 29:767-774.

Russell, C., Mewaldt, R., Luhmann, J., Mason, G.M., von Rosenvigne, T.T., Cohen, C.M.S., Leske, R.A., Bomez-Herrero, R., Klassen, A., Galvin, A.B., and Simunac, K.D.C. (2013) The very unusual interplanetary coronal mass ejection of 2012 July 23: a blast wave mediated by solar energetic particles. The Astrophysical Journal, 770, doi:10.1088/0004-637X/770/1/38.

Scalo, J., Kaltenegger, L., Segura, A.G., Fridlund, M., Ribas, I., Kulikov, Y.N., Grenfell, J.L., Rauer, H., Odert, P., Leitzinger, M., Selsis, F., Khodachenko, M.L., Eiroa, C., Kasting, J., and Lammer, H. (2007) M stars as targets for terrestrial exoplanet searches and biosignature detection. Astrobiology 7:85-166.

Schwieterman, E.W., Meadows, V.S., Domagal-Goldman, S.D., Deming, L.D., Arney, G.N., Luger, R., Harman, C.E., Misra, A., and Barnes, R. (2016) Identifying planetary biosignature impostors: spectral features of $\mathrm{CO}$ and $\mathrm{O}_{4}$ resulting from abiotic $\mathrm{O}_{2} / \mathrm{O}_{3}$ production. Astrophys $J$ 819:L13.

Segura, A., James, F.K., Victoria, M., Martin, C., John, S., David, C., Rebecca, A.H.B., and Giovanna, T. (2005) Biosignatures from Earth-like planets around M dwarfs. Astrobiology 5:706-725.

Segura, A., Krelove, K., Kasting, J.F., Sommerlatt, D., Meadows, V., Crisp, D., Cohen, M., and Mlawer, E. (2003) Ozone concentrations and ultraviolet fluxes on Earth-like planets around other stars. Astrobiology 3:689-708.

Segura, A., Walkowicz, L.M., Meadows, V., Kasting, J., and Hawley, S. (2010) The effect of a strong stellar flare on the atmospheric chemistry of an Earth-like planet orbiting an M dwarf. Astrobiology 10:751-771.

Shea, M.A. and Smart, D.F. (1992) Recent and historical solar proton events. Radiocarbon 34:255-262.

Shields, A.L., Ballard, S., and Johnson, J.A. (2016) The habitability of planets orbiting M-dwarf stars. Phys Rep 663:1-38.

Silvestri, N. M., Hawley, S. L., and Oswalt, T. D. (2005) The chromospheric activity and ages of $\mathrm{M}$ dwarf stars in wide binary systems. Astron J 129, doi:10.1086/429593.

Smart, D., Shea, M., and Flückiger, E. (2000) Magnetospheric models and trajectory computations. In Cosmic Rays and Earth, Springer, Dordrecht, the Netherlands, pp 305-333.

Sukhodolov, T., Usoskin, I., Rozanov, E., Asvestari, E., Ball, W.T., Curran, M.A., Fischer, H., Kovaltsov, G., Miyake, F., Peter, T., Plummer, C., Schmutz, W., Severi, M., and Traversi, 
R. (2017) Atmospheric impacts of the strongest known solar particle storm of 775 AD. Sci Rep 7, doi:10.1038/srep45257.

Tarter, J.C., Backus, P.R., Mancinelli, R.L., Aurnou, J.M., Backman, D.E., Basri, G.S., Boss, A.P., Clarke, A., Deming, D., Doyle, L.R., Feigelson, E.D., Freund, F., Grinspoon, D.H., Haberle, R.M., Hauck, S.A., II, Heath, M.J., Henry, T.J., Hollingsworth, J.L., Joshi, M.M., Kilston, S., Liu, M.C., Meikle, E., Reid, I.N., Rothschild, L.J., Scalo, J., Segura, A., Tang, C.M., Tiedje, J.M., Turnbull, M.C., Walkowicz, L.M., Weber, A.L., and Young, R.E. (2007) A reappraisal of the habitability of planets around M dwarf stars. Astrobiology 7:30-65.

Thomas, B.C. (2018) Photobiological effects at Earth's surface following a 50 pc Supernova. Astrobiology 18:481-490.

Toon, O.B., McKay, C.P., Ackerman, T.P., and Santhanam, K. (1989) Rapid calculation of radiative heating rates and photodissociation rates in inhomogeneous multiple scattering atmospheres. J Geophys Res: Atmospheres 94:16287-16301.

Turbet, M., Leconte, J., Selsis, F., Bolmont, E., Forget, F., Ribas, I., Raymond, S., and Anglada-Escudé, G. (2016) The habitability of Proxima Centauri b II. Possible climates and observability. Astron Astrophys 596:A112.

Vida, K., Kővári, Zs., Pál, A., Oláh, K., and Kriskovics, L. (2017) Frequent flaring in the TRAPPIST-1 system-unsuited for life? Astrophys J 841, doi:10.3847/1538-4357/aa6f05.

Waite, J., Young, D., Cravens, T., Coates, A.J., Crary, F.J., Magee, B., and Westlake J. (2007) Science 316:870-875.

Walkowicz, L.M., Johns-Krull, C.M., and Hawley, S.L. (2008) Characterizing the near-UV environment of M dwarfs. Astrophys $J$ 677, doi:10.1086/526421.

West, A.A., Bochanski, J.J., Hawley, S.L., Cruz, K.L., Covey, K.R., Silvestri, N.M., Reid, I.N., and Liebert, J. (2006) Using the galactic dynamics of M7 dwarfs to infer the evolution of their magnetic activity. Astron J 132:2507-2512.

West, A.A., Hawley, S.L., Bochanski, J.J., Covey, K.R., Reid, I.N., Dhital, S., Hilton, E.J., and Masuda, M. (2008) Constraining the age-activity relation for cool stars: the Sloan Digital Sky Survey Data Release 5 low-mass star spectroscopic sample. Astron J 135:785-795.

Yashiro, S., Akiyama, S., Gopalswamy, N., and Howard, R. (2006) Different power-law indices in the frequency distri- butions of flares with and without coronal mass ejections. Astrophys J 650:L143-L146.

Youngblood, A., France, K., Loyd, R., Brown, A., Mason, J.P., Schneider, P.C., Tilley, M.A., Berta-Thompson, Z.K., Buccino, A., Froning, C.S., Hawley, S.L., Linsky, J., Mauas, P.J.D., Redfield, S., Kowalski, A., Miguel, Y., Newton, E.R., Rugheimer, S., Segura, A., Roberge, A., and Vieytes, M. (2017) The MUSCLES Treasury Survey. IV. Scaling relations for ultraviolet, $\mathrm{Ca}$ II $\mathrm{K}$, and energetic particle fluxes from $\mathrm{M}$ dwarfs. Astrophys $J$ 843, doi:10.3847/1538-4357/aa76dd.

Yung, Y.L. and DeMore, W.B. (1999) Photochemistry of Planetary Atmospheres, Oxford University Press, New York.

Address correspondence to: Matt A. Tilley

University of Washington Johnson Hall Rm-070

Box 351310

Seattle, WA 98195-1310

E-mail: mtilley@uw.edu

Submitted 21 November 2017 Accepted 7 June 2018

$\quad$ Abbreviations Used
AD Leo $=$ AD Leonis
CME $=$ coronal mass ejection
$\mathrm{EM}=$ electromagnetic
FFD $=$ flare frequency distribution
FWHM $=$ full-width half-maximum
JWST $=$ James Webb Space Telescope
LUVOIR $=$ Large UV Optical Infrared Survey Telescope
pfu $=$ proton flux units
RRTM $=$ rapid radiative transfer model
SEPs $=$ stellar energetic particles
TOA $=$ top of the atmosphere

